\title{
An empirically based tool for analyzing mortality associated with congenital heart surgery
}

Sean M. O’Brien, PhD, ${ }^{a}$ David R. Clarke, MD, ${ }^{\mathrm{b}}$ Jeffrey P. Jacobs, MD, ${ }^{\mathrm{c}}$ Marshall L. Jacobs, MD, ${ }^{\mathrm{d}}$ Francois G. Lacour-Gayet, MD, ${ }^{\mathrm{b}}$ Christian Pizarro, MD, ${ }^{\mathrm{e}}$ Karl F. Welke, MD, ${ }^{\mathrm{f}}$ Bohdan Maruszewski, MD, ${ }^{\mathrm{g}}$ Zdzislaw Tobota, MD, ${ }^{\mathrm{h}}$ Weldon J. Miller, MD,${ }^{\mathrm{i}}$ Leslie Hamilton, MD, ${ }^{\mathrm{j}}$ Eric D. Peterson, MD, MPH, ${ }^{\mathrm{a}}$ Constantine Mavroudis, MD, ${ }^{\mathrm{d}}$ and Fred H. Edwards, $\mathrm{MD}^{\mathrm{k}}$

Objective: Analysis of congenital heart surgery results requires a reliable method of estimating the risk of adverse outcomes. Two major systems in current use are based on projections of risk or complexity that were predominantly subjectively derived. Our goal was to create an objective, empirically based index that can be used to identify the statistically estimated risk of in-hospital mortality by procedure and to group procedures into risk categories.

Methods: Mortality risk was estimated for 148 types of operative procedures using data from 77,294 operations entered into the European Association for Cardiothoracic Surgery (EACTS) Congenital Heart Surgery Database (33,360 operations) and the Society of Thoracic Surgeons (STS) Congenital Heart Surgery Database (43,934 patients) between 2002 and 2007. Procedure-specific mortality rate estimates were calculated using a Bayesian model that adjusted for small denominators. Each procedure was assigned a numeric score (the STS-EACTS Congenital Heart Surgery Mortality Score [2009]) ranging from 0.1 to 5.0 based on the estimated mortality rate. Procedures were also sorted by increasing risk and grouped into 5 categories (the STS-EACTS Congenital Heart Surgery Mortality Categories [2009]) that were chosen to be optimal with respect to minimizing within-category variation and maximizing between-category variation. Model performance was subsequently assessed in an independent validation sample $(\mathrm{n}=27,700)$ and compared with 2 existing methods: Risk Adjustment for Congenital Heart Surgery (RACHS-1) categories and Aristotle Basis Complexity scores.

Results: Estimated mortality rates ranged across procedure types from $0.3 \%$ (atrial septal defect repair with patch) to $29.8 \%$ (truncus plus interrupted aortic arch repair). The proposed STS-EACTS score and STS-EACTS categories demonstrated good discrimination for predicting mortality in the validation sample (C-index $=0.784$ and 0.773 , respectively). For procedures with more than 40 occurrences, the Pearson correlation coefficient between a procedure's STS-EACTS score and its actual mortality rate in the validation sample was 0.80 . In the subset of procedures for which RACHS-1 and Aristotle Basic Complexity scores are defined, discrimination was highest for the STSEACTS score $(\mathrm{C}$-index $=0.787)$, followed by STS-EACTS categories $(\mathrm{C}-\mathrm{index}=0.778)$, RACHS-1 categories $(\mathrm{C}$-index $=0.745)$, and Aristotle Basic Complexity scores $(\mathrm{C}$-index $=0.687)$. When patient covariates were added to each model, the C-index improved: STS-EACTS score $(\mathrm{C}$-index $=0.816)$, STS-EACTS categories $(\mathrm{C}$-index $=$ 0.812), RACHS-1 categories $(\mathrm{C}$-index $=0.802)$, and Aristotle Basic Complexity scores $(\mathrm{C}$-index $=0.795)$.

Conclusion: The proposed risk scores and categories have a high degree of discrimination for predicting mortality and represent an improvement over existing consensus-based methods. Risk models incorporating these measures may be used to compare mortality outcomes across institutions with differing case mixes.

Earn CME credits at

http://cme.ctsnetjournals.org

Cardiac surgeons have recognized and emphasized the need to establish clinical registries and quantitative tools for re-

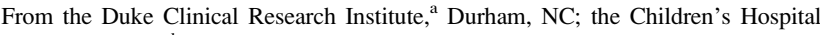
Heart Institute, ${ }^{\mathrm{b}}$ Denver, Colo; the Congenital Heart Institute of Florida (CHIF), ${ }^{\mathrm{c}}$ Saint Petersburg and Tampa, Fla; The Cleveland Clinic, ${ }^{\mathrm{d}}$ Cleveland, Ohio; the Nemours Cardiac Center, ${ }^{\text {e }}$ Alfred I. duPont Hospital for Children, Wilmington, Del; the Oregon Health and Science University, ${ }^{\mathrm{f}}$ Portland, Ore; Memorial Hospital Child's Health Centre, ${ }^{\mathrm{g}}$ Warsaw, Poland; Children's Memorial Health Institute, ${ }^{\mathrm{h}}$ Warsaw, Poland; Rho, Inc, ${ }^{\mathrm{i}}$ Chapel Hill, NC; Freeman Hospital, ${ }^{\mathrm{j}}$ Newcastle upon Tyne, United Kingdom; and the University of Florida, ${ }^{\mathrm{k}}$ Jacksonville, Fla.

Read at the Thirty-fourth Annual Meeting of The Western Thoracic Surgical Association, Kona, Hawaii, June 25-28, 2008.
}

sponsible reporting of outcomes. Large multi-institutional databases, such as the Society of Thoracic Surgeons (STS) Adult Cardiac Surgery Database, among others, have developed, applied, and validated methods of risk adjustment in reporting outcomes. This has addressed appropriate concerns that the reporting of raw, unadjusted mortality data is misleading and potentially penalizes surgeons and centers

Received for publication June 20, 2008; revisions received Nov 18, 2008; accepted for publication March 7, 2009.

Address for reprints: Sean M. O'Brien, PhD, Box 17969, Duke Clinical Research Institute, Durham, NC 27715 (E-mail: obrie027@mc.duke.edu).

J Thorac Cardiovasc Surg 2009; 138:1139-53

$0022-5223 / \$ 36.00$

Copyright (C) 2009 by The American Association for Thoracic Surgery doi:10.1016/j.jtcvs.2009.03.071 


\section{Abbreviations and Acronyms \\ $\mathrm{ABC}=$ Aristotle Basic Complexity \\ EACTS $=$ European Association for \\ Cardiothoracic Surgery \\ RACHS-1 = Risk Adjustment for Congenital Heart Surgery \\ STS = Society of Thoracic Surgeons}

that manage high-risk patients and complex procedures because observed mortality rates might be higher than in centers dealing with less challenging cases. The kinds of statistical tools and risk models that have been developed to address these issues when the clinical substrate is adult patients with acquired cardiovascular disease cannot simply be applied to the population of pediatric and adult patients with congenital heart disease. Here the problem is considerably more complex, in large part because the individual diagnoses and distinct types of surgical procedures number in the hundreds, despite the fact that the universe of patients with congenital heart disease is considerably smaller than that of adult patients with ischemic and valvular heart disease. As a result, the number of patients in some diagnostic and procedural groups is quite small. Nonetheless, it is recognized that the need to establish tools for case-mix adjustment is fundamental to any systematic attempt to measure outcomes, compare performance, and sustain a program of continual quality improvement.

As a response to the need for case-mix adjustment of outcome data but in the absence of significant amounts of registry data in 2000, the Aristotle Complexity score was developed. ${ }^{1,2}$ Using the expert opinions of 50 internationally based surgeons, the Aristotle Basic Complexity (ABC) score was constructed for 145 distinct congenital heart surgery procedures. Three components (potential for mortality, potential for morbidity, and technical difficulty) were subjectively scored, and the sum became the $\mathrm{ABC}$ score.

Separately, another group of researchers developed the Risk Adjustment for Congenital Heart Surgery (RACHS1) system, also using an expert panel. ${ }^{3,4}$ RACHS-1 groups procedures into 6 levels of increasing risk of mortality. This allocation of procedures was subsequently refined using empirical data from 2 multi-institutional registries. When compared with the ABC score, the RACHS- 1 categories appear to have better discrimination for predicting mortality, whereas the $\mathrm{ABC}$ score covers a larger proportion of congenital heart surgery case volume. ${ }^{5-7}$

The largest validation study of the $\mathrm{ABC}$ score was recently conducted by using a combined sample of nearly 36,000 patients from the STS Congenital Heart Surgery Database and the European Association for Cardiothoracic Surgery (EACTS) Congenital Heart Surgery Database. ${ }^{7}$ In that study there was a significant increasing association between the $\mathrm{ABC}$ score and in-hospital mortality, with an overall $\mathrm{C}$-index of 0.70. Although it was clear that the ABC score generally discriminated between low-risk and high-risk procedures, it was also clear that for a relatively small number of individual procedures, the initial estimation of mortality risk by the Aristotle international panel of surgical experts did not accurately predict the actual empirical estimates observed over the ensuing decade.

The goal of the present study was to derive a new system for classifying congenital heart surgery procedures based on their potential for in-hospital mortality using empirical data from the STS and EACTS databases. There were 3 specific objectives.

First, we sought to estimate procedure-specific relative risks of in-hospital mortality using a statistical model that accounts for uncertainty in procedures with small sample sizes.

Second, we sought to convert these procedure-specific mortality estimates into a scale ranging from 0.1 to 5.0. The range of this scale was chosen for consistency with the Aristotle method. The resulting score has been named the STS-EACTS Congenital Heart Surgery Mortality Score (2009) (or, briefly, the STS-EACTS score).

Third, we sought to group procedures with similar estimated mortality risk into a small number of relatively homogeneous categories (the STS-EACTS Congenital Heart Surgery Mortality Categories [2009] or, briefly, the STSEACTS categories). These categories are intended to serve as a stratification variable that can be used to adjust for case mix when analyzing outcomes and comparing institutions.

\section{MATERIALS AND METHODS \\ Study Population}

The STS Congenital Heart Surgery Database and the EACTS Database are described elsewhere. ${ }^{8}$ The study population consisted of patients who underwent a congenital cardiovascular operation at an STS-participating hospital between January 1, 2002, and December 31, 2006, or at an EACTS-participating hospital between January 1, 2002, and April 4, 2007. Data from 1 STS center were excluded because this participant did not consistently report outcomes during the study period. Only the first operation of each hospital admission was analyzed. Operations were included if they involved one of the 148 cardiovascular procedures listed in Table 1. This list includes all cardiovascular procedures that were included in the short-list nomenclature of the STS and EACTS databases and appeared at least once as the primary procedure of an operation in the STS-EACTS dataset. Patients weighing less than or equal to $2500 \mathrm{~g}$ undergoing patent ductus arteriosus ligation as their primary procedure were excluded from the analysis because they are not included in mortality calculations in the EACTS and STS Congenital Database reports. In addition, $244(0.3 \%)$ patients with missing in-hospital mortality status were excluded. The final study population consisted of 43,934 operations from 57 centers in the STS database and 33,360 operations from 91 centers in the EACTS database for a total of 77,294 operations.

The risk tool developed using this dataset was subsequently validated in a separate sample of STS and EACTS patients meeting the same inclusion criteria described above. This validation sample consisted of 20,042 operations performed between January 1, 2007, and June 30, 2008, in the STS database and 7658 operations performed between April 5, 2007, and April 8,2008 , in the EACTS database. 
TABLE 1. Procedure names, proposed scores and categories, and data for model development

\begin{tabular}{|c|c|c|c|c|c|c|c|}
\hline \multirow[b]{2}{*}{ Procedure name } & \multicolumn{3}{|c|}{ Procedure scores } & \multicolumn{2}{|c|}{ No. of operations } & \multicolumn{2}{|c|}{ Estimated mortality risk } \\
\hline & $\begin{array}{c}\text { Difficulty } \\
\text { ranking }\end{array}$ & $\begin{array}{c}\text { Mortality } \\
\text { score }\end{array}$ & $\begin{array}{c}\text { Mortality } \\
\text { category }\end{array}$ & $\begin{array}{c}\text { All } \\
\text { operations }\end{array}$ & $\begin{array}{c}\text { No. with } \\
\text { nonmissing } \\
\text { mortality }\end{array}$ & $\begin{array}{c}\text { Unadjusted } \\
\%(\mathbf{9 5} \% \text { interval } \%) \\
\end{array}$ & $\begin{array}{c}\text { Model based } \\
\% \text { (95\% interval } \dagger) \\
\end{array}$ \\
\hline ASD repair, patch & 8 & 0.1 & 1 & 4035 & 4028 & $0.2 \%(0.1 \%-0.4 \%)$ & $0.3 \%(0.1 \%-0.5 \%)$ \\
\hline $\begin{array}{l}\text { AVC (AVSD) repair, } \\
\text { partial (incomplete) } \\
\text { (PAVSD) }\end{array}$ & 31 & 0.1 & 1 & 1064 & 1062 & $0.3 \%(0.1 \%-0.8 \%)$ & $0.5 \%(0.2 \%-0.9 \%)$ \\
\hline $\begin{array}{l}\text { ASD repair, patch }+ \\
\text { PAPCV repair }\end{array}$ & 28 & 0.2 & 1 & 438 & 438 & $0.2 \%(0.0 \%-1.3 \%)$ & $0.6 \%(0.2 \%-1.4 \%)$ \\
\hline $\begin{array}{l}\text { Aortic stenosis, subvalvar, } \\
\text { repair }\end{array}$ & 42 & 0.2 & 1 & 1834 & 1828 & $0.5 \%(0.3 \%-1.0 \%)$ & $0.6 \%(0.3 \%-1.0 \%)$ \\
\hline ICD (AICD) implantation & 14 & 0.2 & 1 & 391 & 384 & $0.3 \%(0.0 \%-1.4 \%)$ & $0.7 \%(0.2 \%-1.6 \%)$ \\
\hline DCRV repair & 48 & 0.2 & 1 & 467 & 467 & $0.4 \%(0.1 \%-1.5 \%)$ & $0.8 \%(0.2 \%-1.6 \%)$ \\
\hline $\begin{array}{l}\text { ASD repair, primary } \\
\text { closure }\end{array}$ & 7 & 0.2 & 1 & 2230 & 2229 & $0.8 \%(0.5 \%-1.3 \%)$ & $0.9 \%(0.5 \%-1.3 \%)$ \\
\hline VSD repair, patch & 32 & 0.2 & 1 & 6717 & 6702 & $0.9 \%(0.7 \%-1.1 \%)$ & $0.9 \%(0.7 \%-1.1 \%)$ \\
\hline Vascular ring repair & 19 & 0.2 & 1 & 899 & 895 & $0.8 \%(0.3 \%-1.6 \%)$ & $0.9 \%(0.4 \%-1.6 \%)$ \\
\hline $\begin{array}{l}\text { Coarctation repair, end to } \\
\text { end }\end{array}$ & 24 & 0.2 & 1 & 1703 & 1702 & $0.9 \%(0.5 \%-1.5 \%)$ & $1.0 \%(0.6 \%-1.5 \%)$ \\
\hline ICD (AICD) procedure & 15 & 0.2 & 1 & 127 & 126 & $0.0 \%(0.0 \%-2.9 \%)$ & $1.0 \%(0.2 \%-2.9 \%)$ \\
\hline PFO, primary closure & 6 & 0.2 & 1 & 217 & 216 & $0.5 \%(0.0 \%-2.6 \%)$ & $1.1 \%(0.3 \%-2.5 \%)$ \\
\hline AVR, bioprosthetic & 55 & 0.3 & 1 & 101 & 101 & $0.0 \%(0.0 \%-3.6 \%)$ & $1.2 \%(0.2 \%-3.4 \%)$ \\
\hline $\begin{array}{l}\text { VSD repair, primary } \\
\text { closure }\end{array}$ & 30 & 0.3 & 1 & 754 & 752 & $1.1 \%(0.5 \%-2.1 \%)$ & $1.2 \%(0.6 \%-2.1 \%)$ \\
\hline PVR & 44 & 0.3 & 1 & 682 & 680 & $1.2 \%(0.5 \%-2.3 \%)$ & $1.3 \%(0.6 \%-2.3 \%)$ \\
\hline Conduit reoperation & 77 & 0.3 & 1 & 1303 & 1299 & $1.3 \%(0.8 \%-2.1 \%)$ & $1.4 \%(0.8 \%-2.1 \%)$ \\
\hline Pacemaker procedure & 3 & 0.3 & 1 & 1411 & 1408 & $1.3 \%(0.8 \%-2.1 \%)$ & $1.4 \%(0.9 \%-2.1 \%)$ \\
\hline PAPVC repair & 27 & 0.3 & 1 & 481 & 481 & $1.2 \%(0.5 \%-2.7 \%)$ & $1.5 \%(0.7 \%-2.7 \%)$ \\
\hline $\begin{array}{l}\text { TOF repair, } \\
\text { ventriculotomy, } \\
\text { nontransanular patch }\end{array}$ & 62 & 0.3 & 1 & 930 & 928 & $1.4 \%(0.7 \%-2.4 \%)$ & $1.5 \%(0.8 \%-2.4 \%)$ \\
\hline $\begin{array}{l}\text { TOF repair, no } \\
\text { ventriculotomy }\end{array}$ & 81 & 0.3 & 1 & 862 & 860 & $1.4 \%(0.7 \%-2.4 \%)$ & $1.5 \%(0.8 \%-2.3 \%)$ \\
\hline $\begin{array}{l}\text { Glenn (unidirectional } \\
\text { cavopulmonary } \\
\text { anastomosis; } \\
\text { unidirectional Glenn } \\
\text { procedure) }\end{array}$ & 41 & 0.3 & 1 & 65 & 65 & $0.0 \%(0.0 \%-5.5 \%)$ & $1.5 \%(0.2 \%-4.3 \%)$ \\
\hline $\begin{array}{l}\text { AVC (AVSD) repair, } \\
\text { intermediate } \\
\text { (transitional) }\end{array}$ & 33 & 0.3 & 1 & 421 & 420 & $1.4 \%(0.5 \%-3.1 \%)$ & $1.6 \%(0.7 \%-3.0 \%)$ \\
\hline $\begin{array}{l}\text { Coarctation repair, } \\
\text { interposition graft }\end{array}$ & 49 & 0.3 & 1 & 114 & 114 & $0.9 \%(0.0 \%-4.8 \%)$ & $1.7 \%(0.4 \%-4.1 \%)$ \\
\hline $\begin{array}{l}\text { Fontan, TCPC, lateral } \\
\text { tunnel, fenestrated }\end{array}$ & 101 & 0.3 & 1 & 743 & 742 & $1.6 \%(0.8 \%-2.8 \%)$ & $1.7 \%(0.9 \%-2.7 \%)$ \\
\hline $\begin{array}{l}\text { Sinus of Valsalva, } \\
\text { aneurysm repair }\end{array}$ & 61 & 0.3 & 1 & 53 & 53 & $0.0 \%(0.0 \%-6.7 \%)$ & $1.7 \%(0.3 \%-5.2 \%)$ \\
\hline AVR, mechanical & 52 & 0.3 & 1 & 384 & 383 & $1.6 \%(0.6 \%-3.4 \%)$ & $1.7 \%(0.7 \%-3.2 \%)$ \\
\hline PDA closure, surgical & 5 & 0.4 & 2 & 1922 & 1910 & $1.8 \%(1.3 \%-2.5 \%)$ & $1.9 \%(1.3 \%-2.5 \%)$ \\
\hline $\begin{array}{l}\text { PA, reconstruction } \\
\text { (plasty), main (trunk) }\end{array}$ & 25 & 0.4 & 2 & 192 & 191 & $1.6 \%(0.3 \%-4.5 \%)$ & $1.9 \%(0.6 \%-4.0 \%)$ \\
\hline LV to aorta tunnel repair & 90 & 0.4 & 2 & 42 & 42 & $0.0 \%(0.0 \%-8.4 \%)$ & $1.9 \%(0.3 \%-5.9 \%)$ \\
\hline Valvuloplasty, mitral & 76 & 0.4 & 2 & 1751 & 1747 & $1.9 \%(1.3 \%-2.6 \%)$ & $1.9 \%(1.3 \%-2.6 \%)$ \\
\hline Valvuloplasty, aortic & 72 & 0.4 & 2 & 861 & 861 & $1.9 \%(1.1 \%-3.0 \%)$ & $1.9 \%(1.1 \%-2.9 \%)$ \\
\hline 11/2 Ventricular repair & 58 & 0.4 & 2 & 39 & 39 & $0.0 \%(0.0 \%-9.0 \%)$ & $2.0 \%(0.3 \%-6.2 \%)$ \\
\hline
\end{tabular}


TABLE 1. Continued

\begin{tabular}{|c|c|c|c|c|c|c|c|}
\hline \multirow[b]{2}{*}{ Procedure name } & \multicolumn{3}{|c|}{ Procedure scores } & \multicolumn{2}{|c|}{ No. of operations } & \multicolumn{2}{|c|}{ Estimated mortality risk } \\
\hline & $\begin{array}{c}\text { Difficulty } \\
\text { ranking }\end{array}$ & $\begin{array}{l}\text { Mortality } \\
\text { score }\end{array}$ & $\begin{array}{l}\text { Mortality } \\
\text { category }\end{array}$ & $\begin{array}{c}\text { All } \\
\text { operations }\end{array}$ & $\begin{array}{c}\text { No. with } \\
\text { nonmissing } \\
\text { mortality }\end{array}$ & $\begin{array}{c}\text { Unadjusted } \\
\%(\mathbf{9 5} \% \text { interval } *)\end{array}$ & $\begin{array}{c}\text { Model based } \\
\%(\mathbf{9 5} \% \text { interval } \dagger)\end{array}$ \\
\hline $\begin{array}{l}\text { Arrhythmia surgery- } \\
\text { ventricular, surgical } \\
\text { ablation }\end{array}$ & 85 & 0.4 & 2 & 33 & 33 & $0.0 \%(0.0 \%-10.6 \%)$ & $2.2 \%(0.3 \%-6.8 \%)$ \\
\hline $\begin{array}{l}\text { Pacemaker implantation, } \\
\text { permanent }\end{array}$ & 2 & 0.4 & 2 & 1086 & 1077 & $2.1 \%(1.4 \%-3.2 \%)$ & $2.2 \%(1.4 \%-3.1 \%)$ \\
\hline Ross procedure & 127 & 0.4 & 2 & 620 & 617 & $2.1 \%(1.1 \%-3.6 \%)$ & $2.2 \%(1.3 \%-3.4 \%)$ \\
\hline Glenn + PA reconstruction & 71 & 0.4 & 2 & 428 & 426 & $2.1 \%(1.0 \%-4.0 \%)$ & $2.2 \%(1.1 \%-3.8 \%)$ \\
\hline Aortopexy & 4 & 0.4 & 2 & 30 & 30 & $0.0 \%(0.0 \%-11.6 \%)$ & $2.3 \%(0.3 \%-7.3 \%)$ \\
\hline $\begin{array}{l}\text { Fontan, atriopulmonary } \\
\text { connection }\end{array}$ & 94 & 0.4 & 2 & 30 & 30 & $0.0 \%(0.0 \%-11.6 \%)$ & $2.3 \%(0.3 \%-6.9 \%)$ \\
\hline $\begin{array}{l}\text { Bilateral bidirectional } \\
\text { cavopulmonary } \\
\text { anastomosis (bilateral } \\
\text { bidirectional Glenn } \\
\text { procedure) }\end{array}$ & 63 & 0.4 & 2 & 449 & 449 & $2.2 \%(1.1 \%-4.1 \%)$ & $2.4 \%(1.2 \%-3.8 \%)$ \\
\hline $\begin{array}{l}\text { Aortic root replacement, } \\
\text { mechanical }\end{array}$ & 111 & 0.5 & 2 & 145 & 145 & $2.1 \%(0.4 \%-5.9 \%)$ & $2.4 \%(0.7 \%-5.1 \%)$ \\
\hline $\begin{array}{l}\text { Conduit placement, LV to } \\
\text { PA }\end{array}$ & 73 & 0.5 & 2 & 25 & 25 & $0.0 \%(0.0 \%-13.7 \%)$ & $2.4 \%(0.3 \%-7.9 \%)$ \\
\hline $\begin{array}{l}\text { Coarctation repair, end to } \\
\text { end, extended }\end{array}$ & 50 & 0.5 & 2 & 1965 & 1961 & $2.5 \%(1.9 \%-3.3 \%)$ & $2.5 \%(1.9 \%-3.3 \%)$ \\
\hline $\begin{array}{l}\text { Anomalous origin of } \\
\text { coronary artery repair }\end{array}$ & 119 & 0.5 & 2 & 327 & 326 & $2.5 \%(1.1 \%-4.8 \%)$ & $2.6 \%(1.2 \%-4.4 \%)$ \\
\hline RVOT procedure & 40 & 0.5 & 2 & 1591 & 1583 & $2.6 \%(1.9 \%-3.5 \%)$ & $2.6 \%(1.9 \%-3.5 \%)$ \\
\hline Aortic aneurysm repair & 93 & 0.5 & 2 & 322 & 321 & $2.5 \%(1.1 \%-4.9 \%)$ & $2.6 \%(1.3 \%-4.5 \%)$ \\
\hline $\begin{array}{l}\text { Congenitally corrected } \\
\text { TGA repair, VSD } \\
\text { closure }\end{array}$ & 106 & 0.5 & 2 & 21 & 21 & $0.0 \%(0.0 \%-16.1 \%)$ & $2.6 \%(0.3 \%-8.8 \%)$ \\
\hline AP window repair & 35 & 0.5 & 2 & 125 & 125 & $2.4 \%(0.5 \%-6.9 \%)$ & $2.7 \%(0.9 \%-5.6 \%)$ \\
\hline Valvuloplasty, pulmonic & 26 & 0.5 & 2 & 307 & 307 & $2.6 \%(1.1 \%-5.1 \%)$ & $2.7 \%(1.3 \%-4.7 \%)$ \\
\hline $\begin{array}{l}\text { TOF repair, } \\
\text { ventriculotomy, } \\
\text { transannular patch }\end{array}$ & 79 & 0.5 & 2 & 2541 & 2535 & $2.7 \%(2.1 \%-3.4 \%)$ & $2.7 \%(2.1 \%-3.4 \%)$ \\
\hline $\begin{array}{l}\text { Aortic root replacement, } \\
\text { bioprosthetic }\end{array}$ & 120 & 0.5 & 2 & 20 & 20 & $0.0 \%(0.0 \%-16.8 \%)$ & $2.7 \%(0.3 \%-9.3 \%)$ \\
\hline $\begin{array}{l}\text { Bidirectional } \\
\text { cavopulmonary } \\
\text { anastomosis } \\
\text { (bidirectional Glenn } \\
\text { procedure) }\end{array}$ & 43 & 0.5 & 2 & 2502 & 2492 & $2.7 \%(2.1 \%-3.4 \%)$ & $2.7 \%(2.1 \%-3.4 \%)$ \\
\hline $\begin{array}{l}\text { Aortic stenosis, } \\
\text { supravalvar, repair }\end{array}$ & 64 & 0.5 & 2 & 336 & 335 & $2.7 \%(1.2 \%-5.0 \%)$ & $2.8 \%(1.4 \%-4.6 \%)$ \\
\hline Pericardiectomy & 20 & 0.5 & 2 & 48 & 48 & $2.1 \%(0.1 \%-11.1 \%)$ & $2.9 \%(0.5 \%-7.5 \%)$ \\
\hline Conduit placement, other & 75 & 0.5 & 2 & 16 & 16 & $0.0 \%(0.0 \%-20.6 \%)$ & $2.9 \%(0.3 \%-9.8 \%)$ \\
\hline $\begin{array}{l}\text { Aneurysm, ventricular, } \\
\text { left, repair }\end{array}$ & 107 & 0.5 & 2 & 47 & 46 & $2.2 \%(0.1 \%-11.5 \%)$ & $3.0 \%(0.5 \%-7.8 \%)$ \\
\hline $\begin{array}{c}\text { Fontan, TCPC, external } \\
\text { conduit, fenestrated }\end{array}$ & 96 & 0.6 & 2 & 1241 & 1238 & $3.0 \%(2.1 \%-4.1 \%)$ & $3.0 \%(2.1 \%-4.0 \%)$ \\
\hline $\begin{array}{l}\text { Pulmonary artery origin } \\
\text { from ascending aorta } \\
\text { (hemitruncus) repair }\end{array}$ & 89 & 0.6 & 2 & 43 & 43 & $2.3 \%(0.1 \%-12.3 \%)$ & $3.1 \%(0.6 \%-8.2 \%)$ \\
\hline
\end{tabular}


TABLE 1. Continued

\begin{tabular}{|c|c|c|c|c|c|c|c|}
\hline \multirow[b]{2}{*}{ Procedure name } & \multicolumn{3}{|c|}{ Procedure scores } & \multicolumn{2}{|c|}{ No. of operations } & \multicolumn{2}{|c|}{ Estimated mortality risk } \\
\hline & $\begin{array}{c}\text { Difficulty } \\
\text { ranking }\end{array}$ & $\begin{array}{l}\text { Mortality } \\
\text { score }\end{array}$ & $\begin{array}{l}\text { Mortality } \\
\text { category }\end{array}$ & $\begin{array}{c}\text { All } \\
\text { operations }\end{array}$ & $\begin{array}{l}\text { No. with } \\
\text { nonmissing } \\
\text { mortality }\end{array}$ & $\begin{array}{c}\text { Unadjusted } \\
\%(\mathbf{9 5} \% \text { interval } \%)\end{array}$ & $\begin{array}{c}\text { Model based } \\
\%(95 \% \text { interval } \dagger)\end{array}$ \\
\hline $\begin{array}{l}\text { ASD, common atrium } \\
\text { (single atrium), } \\
\text { septation }\end{array}$ & 18 & 0.6 & 2 & 44 & 44 & $2.3 \%(0.1 \%-12.0 \%)$ & $3.1 \%(0.5 \%-8.3 \%)$ \\
\hline PAPVC, scimitar, repair & 91 & 0.6 & 2 & 72 & 72 & $2.8 \%(0.3 \%-9.7 \%)$ & $3.2 \%(0.8 \%-7.7 \%)$ \\
\hline $\begin{array}{l}\text { Fontan, TCPC, external } \\
\text { conduit, nonfenestrated }\end{array}$ & 97 & 0.6 & 2 & 809 & 807 & $3.2 \%(2.1 \%-4.7 \%)$ & $3.2 \%(2.1 \%-4.6 \%)$ \\
\hline $\begin{array}{l}\text { Ligation, pulmonary } \\
\text { artery }\end{array}$ & 16 & 0.6 & 2 & 11 & 11 & $0.0 \%(0.0 \%-28.5 \%)$ & $3.4 \%(0.4 \%-12.1 \%)$ \\
\hline $\begin{array}{l}\text { Coronary artery fistula } \\
\text { ligation }\end{array}$ & 17 & 0.6 & 2 & 39 & 38 & $2.6 \%(0.1 \%-13.8 \%)$ & $3.4 \%(0.6 \%-9.2 \%)$ \\
\hline $\begin{array}{l}\text { Aortic root replacement, } \\
\text { valve sparing }\end{array}$ & 142 & 0.6 & 2 & 37 & 37 & $2.7 \%(0.1 \%-14.2 \%)$ & $3.4 \%(0.6 \%-9.2 \%)$ \\
\hline $\begin{array}{l}\text { Mitral stenosis, } \\
\text { supravalvar mitral ring } \\
\text { repair }\end{array}$ & 74 & 0.6 & 2 & 86 & 86 & $3.5 \%(0.7 \%-9.9 \%)$ & $3.6 \%(1.0 \%-7.7 \%)$ \\
\hline $\begin{array}{l}\text { Arrhythmia surgery-atrial, } \\
\text { surgical ablation }\end{array}$ & 84 & 0.7 & 2 & 273 & 272 & $3.7 \%(1.8 \%-6.7 \%)$ & $3.6 \%(1.9 \%-5.9 \%)$ \\
\hline $\begin{array}{l}\text { Systemic venous stenosis } \\
\text { repair }\end{array}$ & 56 & 0.7 & 2 & 59 & 59 & $3.4 \%(0.4 \%-11.7 \%)$ & $3.7 \%(0.9 \%-8.6 \%)$ \\
\hline $\begin{array}{l}\text { PA, reconstruction } \\
\text { (plasty), branch, } \\
\text { peripheral (at or beyond } \\
\text { the hilar bifurcation) }\end{array}$ & 70 & 0.7 & 2 & 189 & 189 & $3.7 \%(1.5 \%-7.5 \%)$ & $3.7 \%(1.6 \%-6.5 \%)$ \\
\hline Valvuloplasty, tricuspid & 57 & 0.7 & 2 & 1182 & 1178 & $3.7 \%(2.7 \%-5.0 \%)$ & $3.7 \%(2.8 \%-4.9 \%)$ \\
\hline TVR & 65 & 0.7 & 2 & 133 & 133 & $3.8 \%(1.2 \%-8.6 \%)$ & $3.8 \%(1.5 \%-7.3 \%)$ \\
\hline $\begin{array}{l}\text { Valve replacement, } \\
\text { truncal valve }\end{array}$ & 46 & 0.7 & 2 & 8 & 8 & $0.0 \%(0.0 \%-36.9 \%)$ & $3.8 \%(0.4 \%-13.8 \%)$ \\
\hline $\begin{array}{l}\text { Fontan, TCPC, lateral } \\
\text { tunnel, nonfenestrated }\end{array}$ & 99 & 0.7 & 2 & 104 & 104 & $3.8 \%(1.1 \%-9.6 \%)$ & $3.9 \%(1.3 \%-7.9 \%)$ \\
\hline Atrial fenestration closure & 38 & 0.7 & 2 & 29 & 29 & $3.4 \%(0.1 \%-17.8 \%)$ & $3.9 \%(0.7 \%-11.3 \%)$ \\
\hline Cor triatriatum repair & 60 & 0.7 & 2 & 177 & 176 & $4.0 \%(1.6 \%-8.0 \%)$ & $4.0 \%(1.8 \%-7.2 \%)$ \\
\hline VSD, multiple, repair & 113 & 0.7 & 2 & 325 & 324 & $4.0 \%(2.2 \%-6.8 \%)$ & $4.0 \%(2.2 \%-6.3 \%)$ \\
\hline $\begin{array}{l}\text { Atrial baffle procedure } \\
\text { (non-Mustard, non- } \\
\text { Senning) }\end{array}$ & 67 & 0.7 & 2 & 26 & 26 & $3.8 \%(0.1 \%-19.6 \%)$ & $4.0 \%(0.7 \%-11.0 \%)$ \\
\hline $\begin{array}{l}\text { Coarctation repair, } \\
\text { subclavian flap }\end{array}$ & 23 & 0.7 & 2 & 219 & 219 & $4.1 \%(1.9 \%-7.7 \%)$ & $4.1 \%(2.0 \%-6.9 \%)$ \\
\hline $\begin{array}{l}\text { Partial left } \\
\text { ventriculectomy (LV } \\
\text { volume reduction } \\
\text { surgery; Batista) }\end{array}$ & 133 & 0.7 & 2 & 26 & 26 & $3.8 \%(0.1 \%-19.6 \%)$ & $4.1 \%(0.7 \%-11.3 \%)$ \\
\hline $\begin{array}{l}\text { TOF repair, RV-PA } \\
\text { conduit }\end{array}$ & 80 & 0.7 & 2 & 362 & 358 & $4.2 \%(2.4 \%-6.8 \%)$ & $4.2 \%(2.4 \%-6.4 \%)$ \\
\hline Transplantation, lung(s) & 129 & 0.8 & 3 & 94 & 93 & $4.3 \%(1.2 \%-10.6 \%)$ & $4.2 \%(1.4 \%-8.6 \%)$ \\
\hline Occlusion MAPCA(s) & 51 & 0.8 & 3 & 26 & 26 & $3.8 \%(0.1 \%-19.6 \%)$ & $4.2 \%(0.7 \%-12.1 \%)$ \\
\hline $\begin{array}{l}\text { Coarctation repair + VSD } \\
\text { repair }\end{array}$ & 112 & 0.8 & 3 & 329 & 327 & $4.3 \%(2.4 \%-7.1 \%)$ & $4.2 \%(2.4 \%-6.6 \%)$ \\
\hline Konno procedure & 131 & 0.8 & 3 & 162 & 162 & $4.3 \%(1.8 \%-8.7 \%)$ & $4.3 \%(1.9 \%-7.6 \%)$ \\
\hline $\begin{array}{l}\text { Coarctation repair, patch } \\
\text { aortoplasty }\end{array}$ & 22 & 0.8 & 3 & 395 & 393 & $4.3 \%(2.5 \%-6.8 \%)$ & $4.3 \%(2.6 \%-6.5 \%)$ \\
\hline
\end{tabular}




\begin{tabular}{|c|c|c|c|c|c|c|c|}
\hline \multirow[b]{2}{*}{ Procedure name } & \multicolumn{3}{|c|}{ Procedure scores } & \multicolumn{2}{|c|}{ No. of operations } & \multicolumn{2}{|c|}{ Estimated mortality risk } \\
\hline & $\begin{array}{l}\text { Difficulty } \\
\text { ranking }\end{array}$ & $\begin{array}{c}\text { Mortality } \\
\text { score }\end{array}$ & $\begin{array}{l}\text { Mortality } \\
\text { category }\end{array}$ & $\begin{array}{c}\text { All } \\
\text { operations }\end{array}$ & $\begin{array}{l}\text { No. with } \\
\text { nonmissing } \\
\text { mortality }\end{array}$ & $\begin{array}{c}\text { Unadjusted } \\
\%(\mathbf{9 5} \% \text { interval } *)\end{array}$ & $\begin{array}{c}\text { Model based } \\
\%(95 \% \text { interval } \dagger)\end{array}$ \\
\hline $\begin{array}{l}\text { PA, reconstruction } \\
\text { (plasty), branch, central } \\
\text { (within the hilar } \\
\text { bifurcation) }\end{array}$ & 68 & 0.8 & 3 & 646 & 644 & $4.3 \%(2.9 \%-6.2 \%)$ & $4.3 \%(2.9 \%-5.9 \%)$ \\
\hline $\begin{array}{l}\text { Aneurysm, pulmonary } \\
\text { artery, repair }\end{array}$ & 53 & 0.8 & 3 & 23 & 23 & $4.3 \%(0.1 \%-21.9 \%)$ & $4.3 \%(0.8 \%-12.2 \%)$ \\
\hline $\begin{array}{l}\text { Aneurysm, ventricular, } \\
\text { right, repair }\end{array}$ & 86 & 0.8 & 3 & 91 & 91 & $4.4 \%(1.2 \%-10.9 \%)$ & $4.3 \%(1.4 \%-8.8 \%)$ \\
\hline $\begin{array}{l}\text { Ventricular septal } \\
\text { fenestration }\end{array}$ & 45 & 0.8 & 3 & 24 & 24 & $4.2 \%(0.1 \%-21.1 \%)$ & $4.4 \%(0.8 \%-12.4 \%)$ \\
\hline $\begin{array}{l}\text { Shunt, ligation and } \\
\text { takedown }\end{array}$ & 11 & 0.8 & 3 & 65 & 65 & $4.6 \%(1.0 \%-12.9 \%)$ & $4.5 \%(1.3 \%-9.9 \%)$ \\
\hline Hemi-Fontan procedure & 78 & 0.8 & 3 & 262 & 260 & $4.6 \%(2.4 \%-7.9 \%)$ & $4.5 \%(2.4 \%-7.1 \%)$ \\
\hline $\begin{array}{l}\text { AVC (AVSD) repair, } \\
\text { complete }\end{array}$ & 87 & 0.8 & 3 & 2869 & 2860 & $4.6 \%(3.9 \%-5.4 \%)$ & $4.6 \%(3.9 \%-5.4 \%)$ \\
\hline $\begin{array}{l}\text { Anomalous systemic } \\
\text { venous connection } \\
\text { repair }\end{array}$ & 54 & 0.8 & 3 & 166 & 166 & $4.8 \%(2.1 \%-9.3 \%)$ & $4.8 \%(2.2 \%-8.2 \%)$ \\
\hline ASO & 115 & 0.8 & 3 & 2069 & 2068 & $4.8 \%(3.9 \%-5.8 \%)$ & $4.8 \%(3.9 \%-5.7 \%)$ \\
\hline $\begin{array}{l}\text { Valvuloplasty, truncal } \\
\text { valve }\end{array}$ & 59 & 0.8 & 3 & 20 & 20 & $5.0 \%(0.1 \%-24.9 \%)$ & $4.8 \%(0.8 \%-13.5 \%)$ \\
\hline $\begin{array}{l}\text { Fontan, atrioventricular } \\
\text { connection }\end{array}$ & 102 & 0.9 & 3 & 2 & 2 & $0.0 \%(0.0 \%-84.2 \%)$ & $4.9 \%(0.4 \%-20.1 \%)$ \\
\hline $\begin{array}{l}\text { Pulmonary embolectomy, } \\
\text { acute pulmonary } \\
\text { embolus }\end{array}$ & 34 & 0.9 & 3 & 2 & 2 & $0.0 \%(0.0 \%-84.2 \%)$ & $5.0 \%(0.4 \%-19.7 \%)$ \\
\hline ASD partial closure & 10 & 0.9 & 3 & 37 & 37 & $5.4 \%(0.7 \%-18.2 \%)$ & $5.1 \%(1.1 \%-12.7 \%)$ \\
\hline Rastelli operation & 125 & 0.9 & 3 & 333 & 333 & $5.4 \%(3.2 \%-8.4 \%)$ & $5.3 \%(3.2 \%-7.8 \%)$ \\
\hline $\begin{array}{l}\text { Conduit placement, } \\
\text { ventricle to aorta }\end{array}$ & 95 & 0.9 & 3 & 1 & 1 & $0.0 \%(0.0 \%-97.5 \%)$ & $5.3 \%(0.5 \%-21.4 \%)$ \\
\hline AVR, homograft & 110 & 1 & 3 & 30 & 30 & $6.7 \%(0.8 \%-22.1 \%)$ & $5.8 \%(1.3 \%-13.8 \%)$ \\
\hline REV & 126 & 1.1 & 3 & 26 & 26 & $7.7 \%(0.9 \%-25.1 \%)$ & $6.3 \%(1.3 \%-15.5 \%)$ \\
\hline $\begin{array}{l}\text { Pulmonary artery sling } \\
\text { repair }\end{array}$ & 105 & 1.1 & 3 & 88 & 86 & $7.0 \%(2.6 \%-14.6 \%)$ & $6.4 \%(2.5 \%-11.9 \%)$ \\
\hline Mustard procedure & 100 & 1.1 & 3 & 25 & 25 & $8.0 \%(1.0 \%-26.0 \%)$ & $6.4 \%(1.4 \%-15.9 \%)$ \\
\hline $\begin{array}{l}\text { Pulmonary atresia-VSD } \\
\text { (including TOF, PA) } \\
\text { repair }\end{array}$ & 92 & 1.1 & 3 & 289 & 289 & $6.6 \%(4.0 \%-10.1 \%)$ & $6.4 \%(4.0 \%-9.3 \%)$ \\
\hline $\begin{array}{l}\text { Conduit placement, RV to } \\
\text { PA }\end{array}$ & 66 & 1.2 & 3 & 965 & 964 & $6.7 \%(5.2 \%-8.5 \%)$ & $6.7 \%(5.2 \%-8.4 \%)$ \\
\hline Pulmonary embolectomy & 37 & 1.2 & 3 & 9 & 9 & $11.1 \%(0.3 \%-48.2 \%)$ & $7.1 \%(1.0 \%-22.1 \%)$ \\
\hline MVR & 69 & 1.3 & 4 & 637 & 636 & $7.4 \%(5.5 \%-9.7 \%)$ & $7.3 \%(5.4 \%-9.4 \%)$ \\
\hline $\begin{array}{l}\text { Pericardial drainage } \\
\text { procedure }\end{array}$ & 1 & 1.3 & 4 & 258 & 256 & $7.8 \%(4.8 \%-11.8 \%)$ & $7.5 \%(4.7 \%-11.0 \%)$ \\
\hline Aortic arch repair & 82 & 1.4 & 4 & 787 & 782 & $7.9 \%(6.1 \%-10.0 \%)$ & $7.8 \%(6.1 \%-9.8 \%)$ \\
\hline $\begin{array}{l}\text { Fontan revision or } \\
\text { conversion (redo } \\
\text { Fontan procedure) }\end{array}$ & 143 & 1.4 & 4 & 68 & 68 & $8.8 \%(3.3 \%-18.2 \%)$ & $7.9 \%(3.1 \%-14.6 \%)$ \\
\hline DOLV repair & 130 & 1.4 & 4 & 7 & 7 & $14.3 \%(0.4 \%-57.9 \%)$ & $7.9 \%(1.0 \%-24.0 \%)$ \\
\hline $\begin{array}{l}\text { DORV, intraventricular } \\
\text { tunnel repair }\end{array}$ & 132 & 1.4 & 4 & 583 & 582 & $8.1 \%(6.0 \%-10.6 \%)$ & $8.0 \%(6.0 \%-10.3 \%)$ \\
\hline
\end{tabular}


TABLE 1. Continued

\begin{tabular}{|c|c|c|c|c|c|c|c|}
\hline \multirow[b]{2}{*}{ Procedure name } & \multicolumn{3}{|c|}{ Procedure scores } & \multicolumn{2}{|c|}{ No. of operations } & \multicolumn{2}{|c|}{ Estimated mortality risk } \\
\hline & $\begin{array}{c}\text { Difficulty } \\
\text { ranking }\end{array}$ & $\begin{array}{l}\text { Mortality } \\
\text { score }\end{array}$ & $\begin{array}{l}\text { Mortality } \\
\text { category }\end{array}$ & $\begin{array}{c}\text { All } \\
\text { operations }\end{array}$ & $\begin{array}{c}\text { No. with } \\
\text { nonmissing } \\
\text { mortality }\end{array}$ & $\begin{array}{c}\text { Unadjusted } \\
\%(\mathbf{9 5} \% \text { interval } \%)\end{array}$ & $\begin{array}{c}\text { Model based } \\
\%(95 \% \text { interval } \dagger)\end{array}$ \\
\hline $\begin{array}{l}\text { Arterial switch procedure } \\
+ \text { aortic arch repair }\end{array}$ & 136 & 1.4 & 4 & 18 & 18 & $11.1 \%(1.4 \%-34.7 \%)$ & $8.0 \%(1.7 \%-20.6 \%)$ \\
\hline PA debanding & 29 & 1.4 & 4 & 104 & 104 & $8.7 \%(4.0 \%-15.8 \%)$ & $8.0 \%(3.7 \%-13.7 \%)$ \\
\hline ASO and VSD repair & 138 & 1.4 & 4 & 987 & 985 & $8.3 \%(6.7 \%-10.2 \%)$ & $8.2 \%(6.6 \%-10.0 \%)$ \\
\hline Cardiac tumor resection & 88 & 1.4 & 4 & 221 & 220 & $8.6 \%(5.3 \%-13.2 \%)$ & $8.3 \%(5.1 \%-12.2 \%)$ \\
\hline Transplantation, heart & 103 & 1.4 & 4 & 626 & 625 & $8.5 \%(6.4 \%-10.9 \%)$ & $8.4 \%(6.3 \%-10.6 \%)$ \\
\hline Coronary artery bypass & 98 & 1.5 & 4 & 62 & 62 & $9.7 \%(3.6 \%-19.9 \%)$ & $8.5 \%(3.5 \%-16.0 \%)$ \\
\hline $\begin{array}{l}\text { TOF-absent pulmonary } \\
\text { valve repair }\end{array}$ & 109 & 1.5 & 4 & 166 & 165 & $9.1 \%(5.2 \%-14.6 \%)$ & $8.6 \%(5.0 \%-13.1 \%)$ \\
\hline $\begin{array}{l}\text { Valve excision, tricuspid } \\
\text { (without replacement) }\end{array}$ & 13 & 1.5 & 4 & 5 & 5 & $20.0 \%(0.5 \%-71.6 \%)$ & $8.8 \%(1.2 \%-28.1 \%)$ \\
\hline $\begin{array}{l}\text { Shunt, systemic to } \\
\text { pulmonary, MBTS }\end{array}$ & 39 & 1.5 & 4 & 2793 & 2785 & $8.9 \%(7.9 \%-10.1 \%)$ & $8.9 \%(7.9 \%-10.0 \%)$ \\
\hline TOF-AVC (AVSD) repair & 122 & 1.6 & 4 & 145 & 144 & $9.7 \%(5.4 \%-15.8 \%)$ & $9.1 \%(5.0 \%-14.1 \%)$ \\
\hline Ross-Konno procedure & 146 & 1.6 & 4 & 205 & 205 & $9.8 \%(6.1 \%-14.7 \%)$ & $9.4 \%(5.8 \%-13.9 \%)$ \\
\hline Senning procedure & 108 & 1.6 & 4 & 45 & 45 & $11.1 \%(3.7 \%-24.1 \%)$ & $9.4 \%(3.5 \%-18.6 \%)$ \\
\hline Ebstein's repair & 124 & 1.6 & 4 & 65 & 65 & $10.8 \%(4.4 \%-20.9 \%)$ & $9.5 \%(4.0 \%-17.6 \%)$ \\
\hline $\begin{array}{l}\text { Aortic arch repair + VSD } \\
\text { repair }\end{array}$ & 123 & 1.7 & 4 & 339 & 338 & $10.1 \%(7.1 \%-13.8 \%)$ & $9.8 \%(6.9 \%-13.1 \%)$ \\
\hline PA banding & 21 & 1.7 & 4 & 1298 & 1292 & $9.9 \%(8.3 \%-11.7 \%)$ & $9.8 \%(8.3 \%-11.5 \%)$ \\
\hline $\begin{array}{l}\text { Aortic root replacement, } \\
\text { homograft }\end{array}$ & 121 & 1.7 & 4 & 104 & 102 & $10.8 \%(5.5 \%-18.5 \%)$ & $9.9 \%(5.1 \%-16.2 \%)$ \\
\hline $\begin{array}{l}\text { Unifocalization } \\
\text { MAPCA(s) }\end{array}$ & 116 & 1.7 & 4 & 319 & 319 & $10.3 \%(7.2 \%-14.2 \%)$ & $10.0 \%(7.1 \%-13.4 \%)$ \\
\hline Aortic dissection repair & 128 & 1.7 & 4 & 32 & 31 & $12.9 \%(3.6 \%-29.8 \%)$ & $10.0 \%(3.0 \%-21.1 \%)$ \\
\hline $\begin{array}{l}\text { Congenitally corrected } \\
\text { TGA repair, VSD } \\
\text { closure and LV to PA } \\
\text { conduit }\end{array}$ & 135 & 1.7 & 4 & 12 & 12 & $16.7 \%(2.1 \%-48.4 \%)$ & $10.1 \%(2.0 \%-25.9 \%)$ \\
\hline $\begin{array}{l}\text { Pulmonary atresia-VSD- } \\
\text { MAPCA } \\
\text { (pseudotruncus) repair }\end{array}$ & 137 & 1.7 & 4 & 160 & 158 & $10.8 \%(6.4 \%-16.7 \%)$ & $10.2 \%(6.1 \%-15.3 \%)$ \\
\hline VSD creation/enlargement & 83 & 1.8 & 4 & 107 & 106 & $11.3 \%(6.0 \%-18.9 \%)$ & $10.4 \%(5.6 \%-16.6 \%)$ \\
\hline HLHS biventricular repair & 145 & 1.9 & 4 & 64 & 64 & $12.5 \%(5.6 \%-23.2 \%)$ & $10.9 \%(4.8 \%-18.8 \%)$ \\
\hline TAPVC repair & 104 & 1.9 & 4 & 1381 & 1379 & $11.2 \%(9.6 \%-13.0 \%)$ & $11.2 \%(9.5 \%-12.8 \%)$ \\
\hline $\begin{array}{l}\text { Pulmonary venous } \\
\text { stenosis repair }\end{array}$ & 117 & 2 & 4 & 270 & 268 & $11.9 \%(8.3 \%-16.4 \%)$ & $11.4 \%(8.0 \%-15.3 \%)$ \\
\hline $\begin{array}{l}\text { Shunt, systemic to } \\
\text { pulmonary, central } \\
\text { (from aorta or to main } \\
\text { pulmonary artery) }\end{array}$ & 47 & 2.1 & 4 & 663 & 661 & $12.3 \%(9.9 \%-15.0 \%)$ & $12.1 \%(9.7 \%-14.6 \%)$ \\
\hline $\begin{array}{l}\text { Interrupted aortic arch } \\
\text { repair }\end{array}$ & 118 & 2.1 & 4 & 519 & 515 & $12.4 \%(9.7 \%-15.6 \%)$ & $12.2 \%(9.6 \%-15.1 \%)$ \\
\hline $\begin{array}{l}\text { Arterial switch procedure } \\
\text { and VSD repair + aortic } \\
\text { arch repair }\end{array}$ & 144 & 2.4 & 4 & 113 & 113 & $15.0 \%(9.0 \%-23.0 \%)$ & $14.0 \%(8.5 \%-20.5 \%)$ \\
\hline Truncus arteriosus repair & 134 & 2.4 & 4 & 592 & 586 & $14.3 \%(11.6 \%-17.4 \%)$ & $14.1 \%(11.4 \%-16.8 \%)$ \\
\hline ASD creation/enlargement & 9 & 2.5 & 4 & 138 & 136 & $15.4 \%(9.8 \%-22.6 \%)$ & $14.5 \%(9.4 \%-20.9 \%)$ \\
\hline Atrial septal fenestration & 12 & 2.6 & 4 & 18 & 18 & $22.2 \%(6.4 \%-47.6 \%)$ & $15.1 \%(4.5 \%-30.8 \%)$ \\
\hline
\end{tabular}


TABLE 1. Continued

\begin{tabular}{|c|c|c|c|c|c|c|c|}
\hline \multirow[b]{2}{*}{ Procedure name } & \multicolumn{3}{|c|}{ Procedure scores } & \multicolumn{2}{|c|}{ No. of operations } & \multicolumn{2}{|c|}{ Estimated mortality risk } \\
\hline & $\begin{array}{c}\text { Difficulty } \\
\text { ranking }\end{array}$ & $\begin{array}{l}\text { Mortality } \\
\text { score }\end{array}$ & $\begin{array}{l}\text { Mortality } \\
\text { category }\end{array}$ & $\begin{array}{c}\text { All } \\
\text { operations }\end{array}$ & $\begin{array}{c}\text { No. with } \\
\text { nonmissing } \\
\text { mortality }\end{array}$ & $\begin{array}{c}\text { Unadjusted } \\
\%(\mathbf{9 5} \% \text { interval } *)\end{array}$ & $\begin{array}{c}\text { Model based } \\
\%(95 \% \text { interval } \dagger)\end{array}$ \\
\hline $\begin{array}{l}\text { Valve closure, tricuspid } \\
\text { (exclusion, } \\
\text { univentricular } \\
\text { approach) }\end{array}$ & 36 & 2.6 & 4 & 5 & 5 & $40.0 \%(5.3 \%-85.3 \%)$ & $15.6 \%(2.7 \%-41.6 \%)$ \\
\hline $\begin{array}{l}\text { Damus-Kaye-Stansel } \\
\text { procedure (creation of } \\
\text { AP anastomosis } \\
\text { without arch } \\
\text { reconstruction) }\end{array}$ & 114 & 2.9 & 5 & 344 & 343 & $17.5 \%(13.6 \%-21.9 \%)$ & $17.1 \%(13.2 \%-21.5 \%)$ \\
\hline $\begin{array}{l}\text { Transplantation, heart and } \\
\text { lung }\end{array}$ & 141 & 3.2 & 5 & 13 & 13 & $30.8 \%(9.1 \%-61.4 \%)$ & $18.7 \%(5.4 \%-39.8 \%)$ \\
\hline $\begin{array}{l}\text { Congenitally corrected } \\
\text { TGA repair, atrial } \\
\text { switch and Rastelli } \\
\text { operation }\end{array}$ & 139 & 3.2 & 5 & 18 & 18 & $27.8 \%(9.7 \%-53.5 \%)$ & $18.9 \%(6.3 \%-37.2 \%)$ \\
\hline $\begin{array}{l}\text { Congenitally corrected } \\
\text { TGA repair, atrial } \\
\text { switch and ASO } \\
\text { (double switch) }\end{array}$ & 148 & 3.4 & 5 & 32 & 32 & $25.0 \%(11.5 \%-43.4 \%)$ & $20.0 \%(9.1 \%-34.7 \%)$ \\
\hline Norwood procedure & 147 & 4 & 5 & 2383 & 2359 & $23.7 \%(22.0 \%-25.4 \%)$ & $23.6 \%(21.9 \%-25.3 \%)$ \\
\hline Truncus + IAA repair & 140 & 5 & 5 & 43 & 43 & $34.9 \%(21.0 \%-50.9 \%)$ & $29.8 \%(17.7 \%-44.3 \%)$ \\
\hline
\end{tabular}

$A S D$, Atrial septal defect; $A V C$, atrioventricular canal; $A V S D$, atrioventricular septal defect; $P A V S D$, partial atrioventricular septal defect; $P A P V C$, partial anomalous pulmonary venous connection; $I C D$, implantable cardioverter defibrillator; $A I C D$, automatic implantable cardioverter defibrillator; $D C R V$, double-chambered right ventricle; VSD, ventricular septal defect; $P F O$, patent foramen ovale; $A V R$, aortic valve replacement; $P V R$, pulmonary valve replacement; $T O F$, tetralogy of Fallot; $T C P C$, total cavopulmonary connection; $P D A$, patent ductus arteriosus; $P A$, pulmonary artery; $L V$, left ventricle; $R V O T$, right ventricular outflow tract; $T G A$, transposition of the great arteries; $A P$, aortopulmonary; TVR, tricuspid valve replacement; $R V$, right ventricle; $M A P C A$, major aortopulmonary collateral artery; $A S O$, arterial switch operation; $R E V$, réparation à l'étage ventriculaire (REV procedure); $M V R$, mitral valve replacement; $D O L V$, double-outlet left ventricle; MBTS, modified Blalock-Taussig shunt; $H L H S$, hypoplastic left heart syndrome; TAPVC, total anomalous pulmonary venous connection; IAA, interrupted aortic arch. $*$ Denotes $95 \%$ exact binomial confidence interval. $\nmid$ Denotes $95 \%$ Bayesian credible interval.

Hospitals participating in the STS and EACTS registries are required to comply with local regulatory and privacy guidelines. The Duke Clinical Research Institute serves as the data analysis center for the STS database and has an agreement, as well as institutional review board approval, to analyze the aggregate deidentified data for research purposes.

\section{Classification of Multiple-Procedure Operations}

Several procedures listed in Table 1 are actually combinations of 2 or more procedures. These combinations were identified by the Aristotle expert panel because they occur frequently in the STS and EACTS databases and because the complexity of the combination is regarded as being different from the complexity of the component procedures when performed in isolation. For all other operations involving combinations of procedures, the operation was classified according to the most technically complex procedure, as determined by the difficulty component of the 2007 update of the $\mathrm{ABC}$ score. The $\mathrm{ABC}$ score contains some ties and is not defined for 3 of the procedures listed in Table 1. To deal with undefined or tied Aristotle scores, 6 of the study authors independently ranked the difficulty of each procedure listed in Table 1 . Undefined or tied Aristotle scores were adjudicated by assigning the operation to the procedure with the highest average ranking determined by the 6 graders. The difficulty rankings are included in Table 1 so that users of the risk tool will be able to replicate our method of classifying multiple-procedure operations.

\section{End Point}

The study end point was in-hospital mortality, which was defined as death during the same hospitalization as surgery regardless of cause.

\section{Estimation of Procedure-Specific Mortality Rates}

Mortality estimates were calculated by using a Bayesian random effects model that adjusted each procedure's mortality rate based on the size of the denominator. Using a statistical model was considered advantageous because several individual procedures had small denominators, and hence their unadjusted mortality rates were susceptible to chance fluctuations. Unlike conventional methods, random effects models use data from all of the procedures in the database when estimating the probability of mortality for any single procedure. This "borrowing of information" across procedures produces estimates with good statistical properties, including smaller standard errors than conventional estimates. Heuristically, the model-based estimate is a weighted average of a procedure's actual observed mortality rate and the overall average mortality rate for all procedures in the database. The model weights an individual procedure's own data more heavily when the denominator is large enough to be reliable and weights the overall average mortality rate more heavily when the denominator is too small to support a reliable mortality estimate. For procedures with more than 200 occurrences, the model-based estimates were virtually identical to the usual unadjusted (raw) mortality percentages (Appendix 1). 


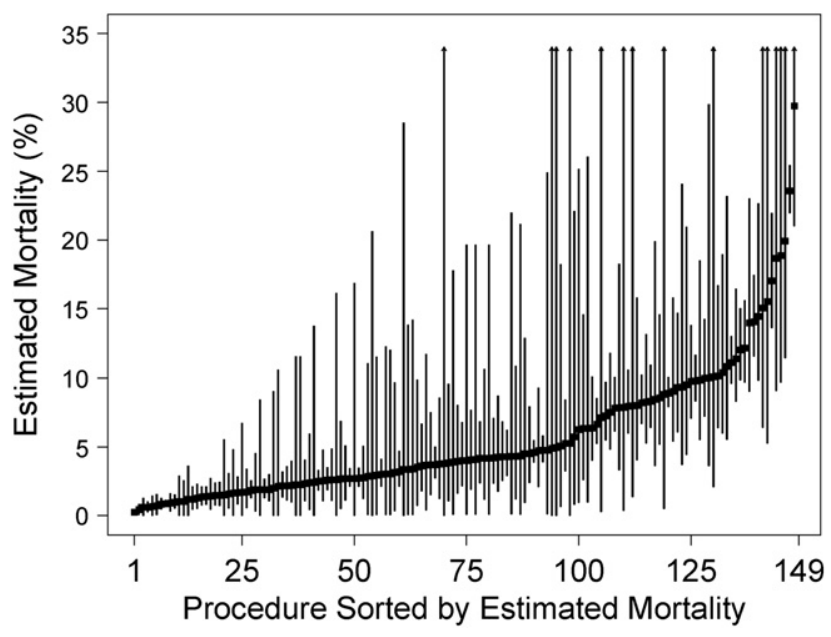

FIGURE 1. Procedure-specific estimated mortality rates. Square dots represent model-based procedure-specific mortality estimates. Vertical lines represent exact $95 \%$ binomial confidence intervals.

\section{Creation of the Mortality Score}

Each procedure was assigned a numeric score (STS-EACTS score) ranging from 0.1 to 5.0. The scores were assigned by shifting and rescaling the estimated procedure-specific mortality rates to lie in the interval from 0.1 to 5.0 and then rounding to one decimal place. The following formula was used:

$$
\text { Mortality score of } j \text {-th procedure }=0.1+4.9 \times \frac{p_{j}-\min }{\max -\min },
$$

where $p_{j}$ denotes the estimated risk of the $j$-th procedure, and $\max$ and $\min$ denote the maximum and minimum values of $p_{j}$ across the 148 procedures.

\section{Creation of Mortality Categories}

Procedures were sorted by increasing estimated risk and partitioned into 5 relatively homogeneous categories (STS-EACTS categories). Five categories was the smallest number that did not result in excessive within-category heterogeneity. Within-category homogeneity was measured objectively using a weighted sum of squares criterion (Appendix 2). ${ }^{9}$ A dynamic programming algorithm was then used to find the categorization that maximizes the homogeneity criterion. This data-driven approach ensures that procedures in the same category will be as similar as possible with respect to their estimated mortality risk

To determine the number of categories, we evaluated the performance of different categorizations consisting of 2 to 20 categories. Performance was assessed internally based on 2 criteria. First, we evaluated the internal homogeneity of the categories using the criterion described in Appendix 2. Second, we assessed the discrimination of the categories as predictors of

TABLE 2. Characteristics of proposed risk categories in 2002-2007 STS and EACTS data

\begin{tabular}{lccccc}
\hline & \multicolumn{5}{c}{ STS-EACTS mortality category } \\
\cline { 2 - 6 } & $\mathbf{1}$ & $\mathbf{2}$ & $\mathbf{3}$ & $\mathbf{4}$ & $\mathbf{5}$ \\
\hline Range of scores & $0.1-0.3$ & $0.4-0.7$ & $0.8-1.2$ & $1.3-2.6$ & $2.7-5.0$ \\
No. of procedures & 26 & 52 & 27 & 37 & 6 \\
No. of patients & 28,363 & 23,235 & 9026 & 13,862 & 2808 \\
No. of deaths & 234 & 601 & 449 & 1374 & 650 \\
Mortality & $0.8 \%$ & $2.6 \%$ & $5.0 \%$ & $9.9 \%$ & $23.1 \%$ \\
\hline
\end{tabular}

STS-EACTS, Society of Thoracic Surgeons-European Association for Cardiothoracic Surgery.
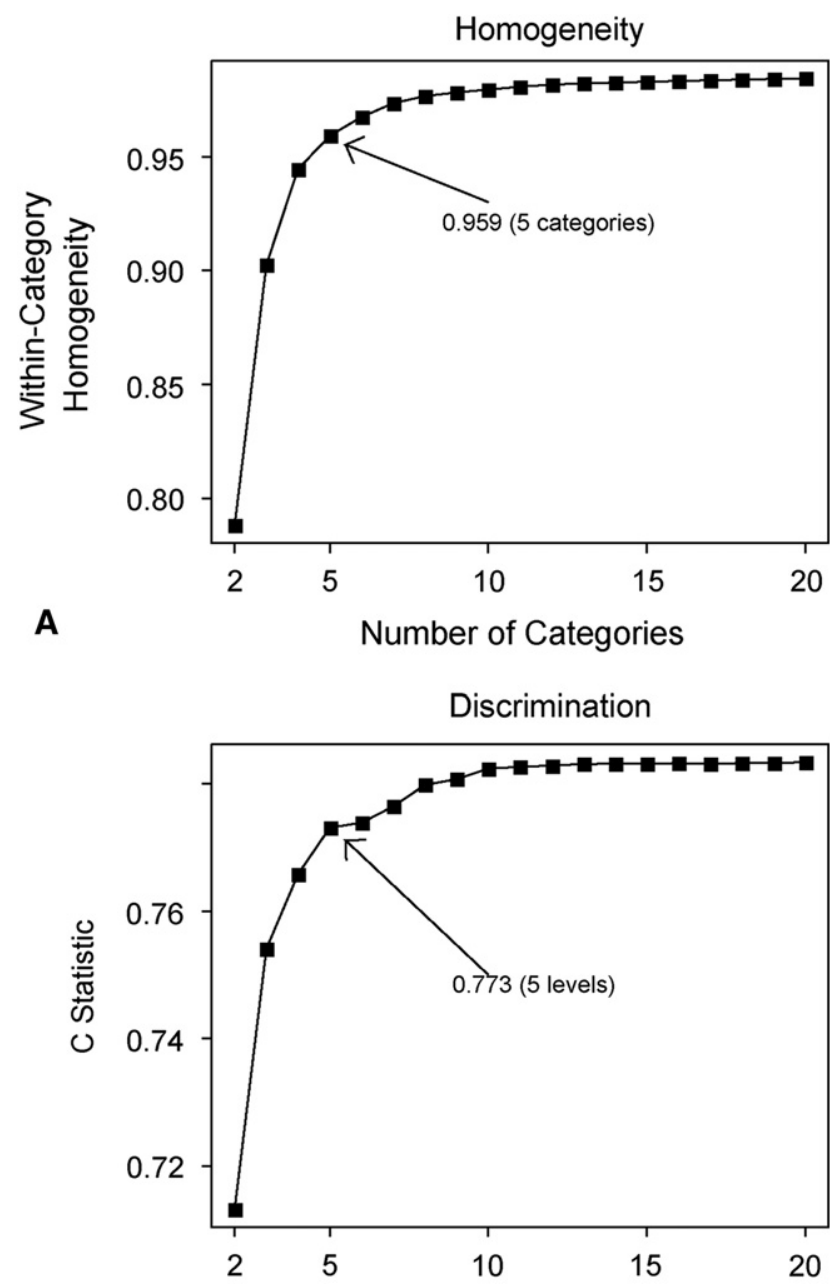

B

Number of Categories

FIGURE 2. Association between number of procedure categories and withincategory homogeneity of mortality risk (Panel A) and discrimination for predicting mortality (Panel B). Performance improves with increasing numbers of categories. See Appendix 2 for definition of within-category homogeneity.

mortality. Discrimination was quantified by the area under the receiver operating characteristic curve (also known as the $\mathrm{C}$-index). ${ }^{10}$ The $\mathrm{C}$-index is interpreted as the probability that a randomly selected patient who died was considered to be higher risk than a randomly selected patient who survived. The C-index generally ranges from 0.5 to 1.0 , with 0.5 representing no discrimination (ie, a coin flip) and 1.0 representing perfect discrimination.

\section{Models Combining Scores and Categories With Patient-Level Risk Factors}

Two logistic regression models were developed to illustrate the utility of modeling the proposed scores and categories together with patient-level risk factors. The first model included the STS-EACTS score (modeled as a continuous variable) plus 3 patient-level factors: age, weight, and preoperative length of stay. To allow for possible nonlinear effects, the score and the square of the score were both entered in the model. Age and weight were modeled jointly by converting them into a single categorical variable with 7 levels (see Results). Preoperative length of stay was dichotomized as less than or equal to 2 days versus more than 2 days. The second model was identical but used the STS-EACTS categories 
TABLE 3. Summary of logistic regression models combining the proposed STS-EACTS scores and categories with patient-level risk factors

\begin{tabular}{|c|c|c|}
\hline \multirow[b]{2}{*}{ Variable } & \multicolumn{2}{|c|}{ Odds ratio $(95 \%$ confidence interval) } \\
\hline & $\begin{array}{l}\text { Model 1: STS- } \\
\text { EACTS score + } \\
\text { patient factors }\end{array}$ & $\begin{array}{c}\text { Model 2: STS- } \\
\text { EACTS categories }+ \\
\text { patient factors }\end{array}$ \\
\hline \multicolumn{3}{|l|}{$\begin{array}{l}\text { STS-EACTS mortality } \\
\text { score }\end{array}$} \\
\hline 0.5 vs 0.25 & $1.4(1.4-1.5)$ & - \\
\hline 1.0 vs 0.25 & $2.6(2.4-2.8)$ & - \\
\hline 2.0 vs 0.25 & $6.3(5.6-7.1)$ & - \\
\hline 4.0 vs 0.25 & $9.4(8.2-10.8)$ & - \\
\hline \multicolumn{3}{|l|}{$\begin{array}{l}\text { STS-EACTS mortality } \\
\text { category }\end{array}$} \\
\hline Category 1 & - & Reference \\
\hline Category 2 & - & $2.9(2.4-3.3)$ \\
\hline Category 3 & - & $4.3(3.6-5.0)$ \\
\hline Category 4 & - & $7.5(6.5-8.7)$ \\
\hline Category 5 & - & $15.9(13.3-18.9)$ \\
\hline \multicolumn{3}{|l|}{$\begin{array}{l}\text { Age and weight } \\
\text { category }\end{array}$} \\
\hline Age $\geq 1$ y & Reference & Reference \\
\hline $\begin{array}{l}\text { Age } 1-11 \mathrm{mo}, \\
\quad \text { weight } \geq 6.0 \mathrm{~kg}\end{array}$ & $1.0(0.8-1.2)$ & $0.9(0.8-1.1)$ \\
\hline $\begin{array}{l}\text { Age } 1-11 \mathrm{mo}, \\
\text { weight } 4.0-5.9 \mathrm{~kg}\end{array}$ & $1.4(1.2-1.6)$ & $1.3(1.2-1.5)$ \\
\hline $\begin{array}{l}\text { Age } 1-11 \mathrm{mo}, \\
\text { weight }<4.0 \mathrm{~kg}\end{array}$ & $2.6(2.2-3.0)$ & $2.6(2.3-3.0)$ \\
\hline $\begin{aligned} & \text { Age }<1 \mathrm{mo}, \text { weight } \\
& \geq 3.0 \mathrm{~kg}\end{aligned}$ & $2.0(1.8-2.2)$ & $1.9(1.7-2.2)$ \\
\hline $\begin{array}{c}\text { Age }<1 \mathrm{mo}, \text { weight } \\
2.0-2.9 \mathrm{~kg}\end{array}$ & $3.3(2.8-3.8)$ & $3.2(2.8-3.7)$ \\
\hline $\begin{array}{l}\text { Age }<1 \mathrm{mo} \text {, weight } \\
\quad<2.0 \mathrm{~kg}\end{array}$ & $4.9(4.2-5.8)$ & $4.9(4.2-5.7)$ \\
\hline \multicolumn{3}{|l|}{ Preoperative LOS } \\
\hline$\leq 2 \mathrm{~d}$ & Reference & Reference \\
\hline$>2 \mathrm{~d}$ & $1.4(1.3-1.6)$ & $1.4(1.3-1.5)$ \\
\hline
\end{tabular}

STS-EACTS, Society of Thoracic Surgeons-European Association for Cardiothoracic Surgery; $L O S$, length of stay.

(modeled as a set of category indicators) instead of the STS-EACTS score. Additional patient factors, such as comorbidities, were not included because these data were not available to us for the EACTS subset at the time of analysis.

\section{Comparisons With RACHS-1 Categories and ABC Scores}

The models described above were also estimated with RACHS- 1 categories in place of the STS-EACTS categories and with the ABC score in place of the STS-EACTS score to facilitate comparisons with existing methods. Briefly, the $\mathrm{ABC}$ score of a procedure is a number ranging from 1.5 to 15 points that reflects the Aristotle expert panel's assessment of that type of procedure's potential for mortality, morbidity, and technical difficulty. When analyzing operations with multiple procedures, the $\mathrm{ABC}$ score was defined as the maximum $\mathrm{ABC}$ score across all procedures in the operation. The RACHS-1 methodology divides procedures into 6 categories based on an expert panel's assessment of the procedure's average

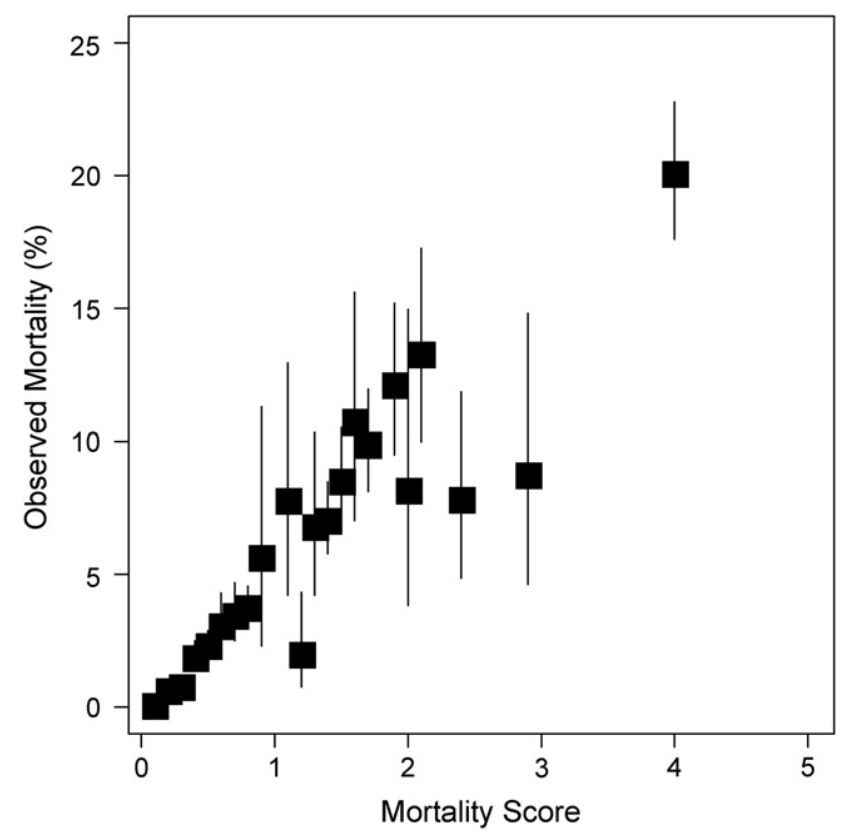

FIGURE 3. Association between Society of Thoracic Surgeons-European Association for Cardiothoracic Surgery score and in-hospital mortality in the validation sample. Square dots represent the aggregate mortality rate of procedures sharing the same risk score. Data points with fewer than 40 observations were excluded from the figure. Vertical lines represent $95 \%$ binomial confidence intervals.

mortality risk, where category 1 has the lowest risk of mortality and category 6 has the highest. Unlike the ABC method, the classification of some procedures is allowed to depend on the patient's age. When analyzing operations with multiple procedures, the operation is assigned to the procedure with the highest RACHS-1 category. Because very few data points

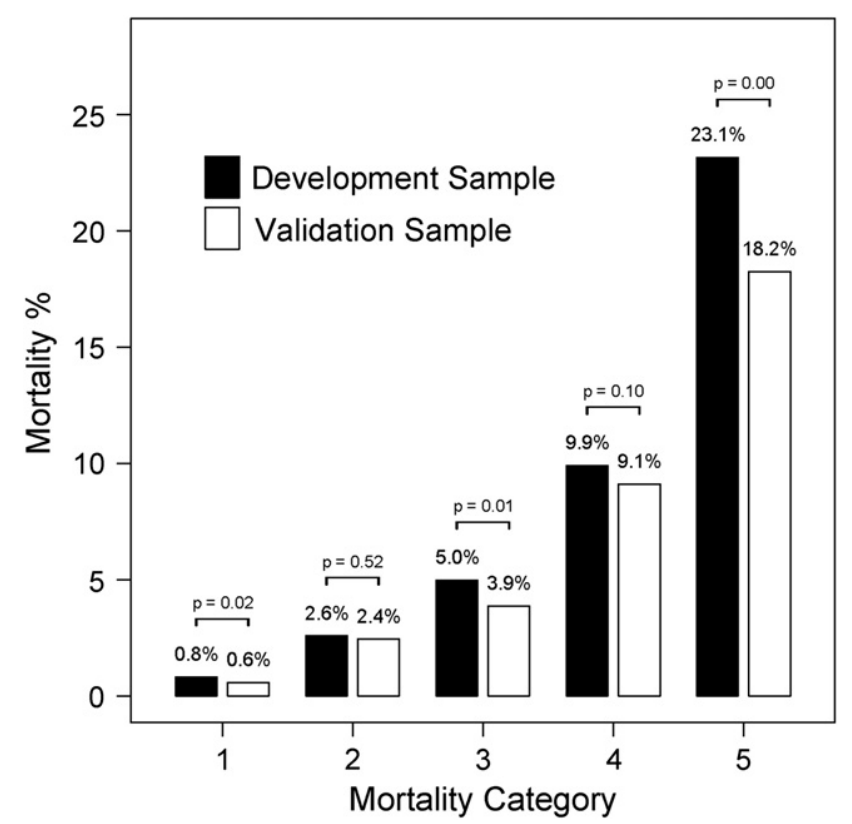

FIGURE 4. Association between proposed risk categories and observed in-hospital mortality. 

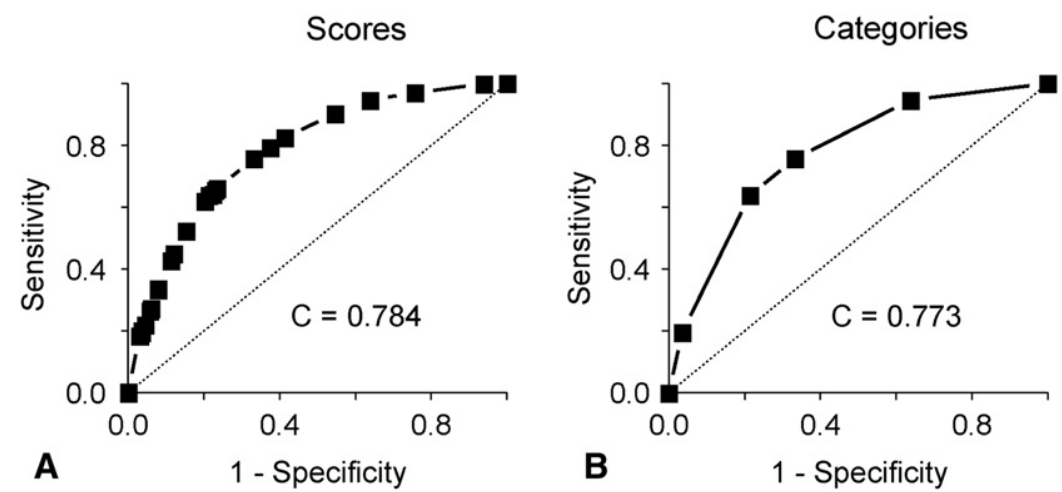

FIGURE 5. Receiver operating characteristic curves for the Society of Thoracic Surgeons-European Association for Cardiothoracic Surgery scores (A) and categories (B) as predictors of in-hospital mortality in the validation sample. The diagonal line is provided as a reference. It is the receiver operating characteristic curve that would be observed hypothetically if the scores and categories were not associated with mortality.

were available in RACHS-1 category 5 , it was combined with category 6 for analysis. The "full" RACHS-1 methodology involves fitting a logistic regression model that includes indicator variables for the RACHS-1 categories together with an indicator variable for single versus multiple cardiac procedures, plus additional adjustment for 3 patient-level risk factors: age, prematurity, and presence of a major noncardiac structural anomaly. Because the required patient-level risk factors were not available in our dataset, we did not implement the full RACHS-1 methodology but instead focused on evaluating the discrimination of the RACHS-1 categories with and without adjustment for patient age, weight, and preoperative length of stay.

\section{Independent Validation Using 2007-2008 Data}

The performance of each model was assessed in a separate, more contemporary sample of STS and EACTS data. Overall discrimination was quantified by the C-index. The ability of the proposed score to predict the risk of individual procedures was quantified by calculating the Pearson correlation coefficient between the score and the actual calculated procedurespecific mortality rate in the validation sample. Because sampling variation in the validation sample might artificially increase or decrease the Pearson correlation coefficient, procedures with fewer than 40 occurrences in the validation sample were excluded when calculating the Pearson correlation coefficient. For graphing the association between the proposed score and observed mortality, data from procedures with the same score were aggregated, and the mortality rate of each group of procedures was plotted as a function of the score, excluding groups with fewer than 40 cases. The entire validation was also repeated in the subset of procedures having at least 200 cases in the development sample. Finally, to permit a fair comparison with RACHS-1 and ABC scores, the performance of each model was assessed in the subset of procedures for which both RACHS-1 categories

TABLE 4. Comparison of C-index for models using the STS-EACTS score, STS-EACTS categories, RACHS-1 categories, and ABC scores*

\begin{tabular}{lcc}
\hline $\begin{array}{c}\text { Method of modeling } \\
\text { procedures }\end{array}$ & $\begin{array}{c}\text { Model without } \\
\text { patient covariates } \\
\text { (C-index) }\end{array}$ & $\begin{array}{c}\text { Model with } \\
\text { patient covariates } \\
\text { (C-index) }\end{array}$ \\
\hline STS-EACTS score & 0.787 & 0.816 \\
STS-EACTS categories & 0.778 & 0.812 \\
RACHS-1 categories & 0.745 & 0.802 \\
ABC score & 0.687 & 0.795 \\
\hline
\end{tabular}

*Validation sample, subset of procedures for which both RACHS-1 categories and $\mathrm{ABC}$ scores are defined. STS-EACTS, Society of Thoracic Surgeons-European Association for Cardiothoracic Surgery; RACHS-1, Risk Adjustment for Congenital Heart Surgery; $A B C$, Aristotle Basic Complexity. and $\mathrm{ABC}$ scores are defined ( $\mathrm{n}=25,106$ patient operations). Statistical comparisons of the $\mathrm{C}$-index for different models were performed using the method of DeLong and colleagues. ${ }^{11}$

\section{RESULTS}

A total of 77,294 patient operations were analyzed, including $3308(4.3 \%)$ in-hospital deaths. There were 71 procedures with at least 200 occurrences, 104 procedures with at least 50 occurrences, and 133 procedures with at least 20 occurrences. Procedures with at least 200 occurrences accounted for $94 \%$ of the total patients and $91 \%$ of the deaths.

\section{Mortality Rates for Individual Procedures}

The frequency of in-hospital mortality for individual procedures ranged from $0 \%$ to $40.0 \%$. There were 18 procedures with zero deaths; all of these had sample sizes smaller than 200. When Bayesian modeling was used to estimate mortality risk for individual procedures, the estimates ranged from $0.3 \%$ (atrial septal defect repair with patch) to $29.8 \%$ (truncus plus interrupted aortic arch repair, Figure 1). For the procedures with more than 200 cases, the raw and model-based estimates were virtually identical (Pearson correlation coefficient $>0.999$, Appendix 1).

\section{Mortality Scores and Categories}

Names of the procedures analyzed in this study are listed in Table 1, along with their raw and model-based mortality estimates and their proposed scores and categories. The STS-EACTS score takes on values between 0.1 and 5.0 and has 29 unique values. The STS-EACTS categories consist of 5 groups labeled 1 to 5 , with higher numbers implying higher mortality risk. The number of patients and procedures per category and their aggregated mortality rates are summarized in Table 2.

The within-category homogeneity criterion and the Cindex were plotted as functions of the number of categories to help us determine the optimal number of mortality categories. As shown in Figure 2, A, within-category 
homogeneity increases rapidly with the number of categories when the number of categories is small. With more than 4 or 5 categories, the homogeneity continues to increase, but the marginal improvement per additional category approaches zero. Similarly, Figure $2, B$, shows that the estimated discrimination of the categories changes dramatically when the number of groups is varied between 2 and 5 , but using more than 5 categories has a relatively modest effect on the C-index. Five categories were chosen as the smallest number that produces both acceptable within-category homogeneity and good discrimination.

Examples of regression models using the proposed scores and categories are summarized in Table 3 . The $\mathrm{C}$-index was 0.814 for the model that combined patient factors with the STS-EACTS score and 0.810 for the model that combined patient factors with the STS-EACTS categories. For comparison, when age, weight, and preoperative length of stay were analyzed in a logistic regression model without adjustment for the STS-EACTS scores or categories, the C-index was 0.755 .

\section{Validation Using 2007-2008 Data}

There was a strong positive association between the proposed STS-EACTS score and actual observed mortality in the validation sample $(\mathrm{C}$-index $=0.784)$. For the 82 procedures with at least 40 occurrences in the validation sample, the Pearson correlation coefficient between the score of a procedure and its actual observed mortality rate in the validation sample was 0.80 . An increasing association between the score and mortality was observed across the range of scores, although several groups of procedures had lower than expected mortality (Figure 3 ).

The observed mortality rate in the validation sample was slightly lower than in the development sample $(3.9 \%$ vs $4.3 \%, P=.004)$, reflecting a trend toward lower mortality in a more contemporary sample. This lower mortality was seen in each of the 5 STS-EACTS categories (Figure 4). Despite the trend toward lower absolute mortality in 2007-2008, the chosen categories continued to perform well at discriminating between high-risk and low-risk procedures $(\mathrm{C}$-index $=0.773$ ). Receiver operating characteristic curves for the proposed scores and categories are displayed in Figure 5. When the validation was repeated in the subset of 73 procedures with at least 200 cases in the development sample, there was a similarly high level of discrimination (C-index $=0.790$ for STS-EACTS scores; C-index $=0.782$ for STS-EACTS categories) and high correlation between the STS-EACTS score and procedure-specific mortality rates (Pearson correlation coefficient $=0.87$ ).

To assess whether the proposed method discriminates mortality better than the existing RACHS- 1 categories and Aristotle scores, each of these was evaluated in the validation sample using the subset of procedures for which both
RACHS- 1 categories and ABC scores are defined. As summarized in Table 4, discrimination was highest for the STS-EACTS score $(C$-index $=0.787$ ), followed by the STS-EACTS categories (C-index $=0.778)$, RACHS-1 categories $(\mathrm{C}$-index $=0.745)$, and $\mathrm{ABC}$ scores $(\mathrm{C}$-index $=$ 0.687 , all differences $P<.0001$ ). Adding patient-level covariates substantially improved each model's discrimination. With the addition of these patient variables, discrimination was highest for the STS-EACTS score $(\mathrm{C}$-index $=0.816)$, followed by STS-EACTS categories $(\mathrm{C}$-index $=0.812$; comparison with STS-EACTS score, $P=.035$ ), RACHS1 categories $(\mathrm{C}$-index $=0.802$; comparison vs STS-EACTS categories, $P=.008$ ), and ABC scores (C-index $=0.795$; comparison vs STS-EACTS score, $P<.0001)$.

\section{DISCUSSION}

The goal of this study was to derive a valid tool that can be used to stratify congenital heart surgery procedures based on their relative risk of in-hospital mortality. Using the combined resources of the STS and EACTS databases, we estimated the average mortality rate of 148 procedures and then applied a data-driven algorithm to determine the grouping of procedures that was optimal in the sense of creating internally homogeneous strata. The resulting scores and categories are intended to serve as tools for case-mix adjustment when comparing outcomes of hospitals that perform congenital heart surgery. These measures can be used to perform a stratified analysis that adjusts for type of procedure or they can be included along with patient-level variables in a comprehensive risk adjustment model.

Previous investigators have used a combination of expert opinion and empirical data to group procedures with a similar risk of in-hospital mortality. Experts initially used clinical judgment to group procedures with a similar potential for in-hospital mortality to create the RACHS-1 risk categories. This allocation of procedures was subsequently refined by using empirical data from 2 multi-institutional registries. The goals of the present study were similar to those of RACHS-1 in that we also sought to create internally homogeneous procedure categories using the end point of discharge mortality. A major difference between our approach and the derivation of RAHCS-1 categories is that our procedure categories were determined empirically without the input of an expert panel. When the proposed methodology was assessed in an independent validation sample, models based on the STS-EACTS score and categories had substantially better discrimination than comparable models based on RACHS-1 categories and ABC scores.

Despite the advantages of an empirically based risk stratification system, there are several limitations and caveats.

First, our study focused on estimating procedural mortality and determining homogeneous procedure categories. Additional research is needed to determine the best method of 
combining these procedural variables with adjustment for patient-specific risk factors.

Second, despite the large database, several individual procedures had small sample sizes, and the true mortality of these procedures may have been estimated with error. We attempted to minimize this error by using a statistical model, which accounted for small denominators.

Third, because the EACTS and STS registries are voluntary, it is possible that the results observed in this database will differ from those of other nonparticipating institutions.

Fourth, because auditing of the STS and EACTS databases has been limited to a small number of sites, the completeness and accuracy of the data are largely unknown. In an audit of 200 patient records from 10 different STS centers, there was $99.0 \%$ agreement in the reporting of discharge mortality by STS sites versus independent auditors and no evidence of selective reporting based on discharge mortality status (personal communication, unpublished STS data).

Another potential limitation rests in the fact that mortality was determined only on the basis of status at the time of discharge. Operative mortality has been defined by the STS Congenital Database Taskforce and the Joint STSEACTS Congenital Database Committee. ${ }^{12}$ It requires knowledge not only of status at discharge but of patient status at 30 days after the operation. Going forward, validation of the STS-EACTS scores and categories using this definition will be possible as the completeness of these data fields in the STS and EACTS databases improves (Appendix 3).

In summary, we have developed a new tool for grouping procedures with a similar empirically estimated risk of inhospital mortality. Empirically based mortality stratification was possible to a considerable extent because of the large sample sizes of the STS and EACTS congenital databases.
The resulting scores and categories can be incorporated into case-mix adjustment methods, such as stratification and regression analysis, to compare institutions on a level playing field.

\section{References}

1. Lacour-Gayet F, Clarke D, Jacobs J, Comas J, Daebritz S, Daenen W, et al. The Aristotle score: a complexity-adjusted method to evaluate surgical results. Eur J Cardiothorac Surg. 2004;25:911-24.

2. Lacour-Gayet F, Clarke D, Jacobs J, Gaynor W, Hamilton L, Jacobs M, et al. The Aristotle score for congenital heart surgery. Semin Thorac Cardiovasc Surg Pediatr Card Surg Annu. 2004;7:185-91.

3. Jenkins KJ. Risk adjustment for congenital heart surgery: the RACHS-1 method. Semin Thorac Cardiovasc Surg Pediatr Card Surg Annu. 2004;7:180-4.

4. Jenkins KJ, Gauvreau K. Center-specific differences in mortality: preliminary analyses using the Risk Adjustment in Congenital Heart Surgery (RACHS-1) method. J Thorac Cardiovasc Surg. 2002;124:97-104.

5. Al-Radi OO, Harrell FE Jr, Caldarone CA, McCrindle BW, Jacobs JP Williams MG, et al. Case complexity scores in congenital heart surgery: a comparative study of the Aristotle Basic Complexity score and the Risk Adjustment in Congenital Heart Surgery (RACHS-1) system. J Thorac Cardiovasc Surg. 2007; 133:865-75.

6. Kang N, Tsang VT, Elliott MJ, de Leval MR, Cole TJ. Does the Aristotle score predict outcome in congenital heart surgery? Eur J Cardiothorac Surg. 2006; 29:986-8.

7. O'Brien SM, Jacobs JP, Clarke DR, Maruszewski B, Jacobs ML, Walters HL 3rd, et al. Accuracy of the Aristotle Basic Complexity score for classifying the mortality and morbidity potential of congenital heart surgery operations. Ann Thorac Surg. 2007;84:2027-37.

8. Jacobs JP, Jacobs ML, Maruszewski B, Lacour-Gayet FG, Clarke DR Tchervenkov CI, et al. Current status of the European Association for CardioThoracic Surgery and the Society of Thoracic Surgeons Congenital Heart Surgery Database. Ann Thorac Surg. 2005;80:2278-84.

9. O'Brien SM. Cutpoint selection for categorizing a continuous predictor. Biometrics. 2004;60:504-9.

10. Hanley JA, McNeil BJ. The meaning and use of the area under a receiver operating characteristic (ROC) curve. Radiology. 1982;143:29-36.

11. DeLong ER, DeLong DM, Clarke-Pearson DL. Comparing the areas under two or more correlated receiver operating characteristic curves: a nonparametric approach. Biometrics. 1988;44:837-45.

12. Jacobs JP, Mavroudis C, Jacobs ML, Maruszewski B, Tchervenkov CI, LacourGayet FG, et al. What is operative mortality? Defining death in a surgical registry database: a report of the STS Congenital Database Taskforce and the Join EACTS-STS Congenital Database Committee. Ann Thorac Surg. 2006;81: $1937-41$.

\section{Appendix 1. Statistical Model for Estimating Procedure-Specific Mortality Rates}

Procedure-specific mortality rates were estimated by using a hierachical (random effects) model. For each of the 148 procedures in the analysis, the number of deaths was modeled by using the following binomial distribution:

$$
y_{j} \sim \operatorname{Binomial}\left(n_{j}, \pi_{j}\right), j=1,2, \ldots, 148,
$$

where $\pi_{j}$ denotes the unknown theoretical probability of mortality for the $j$-th procedure, $n_{j}$ denotes the number of patients undergoing the procedure in the database (denominator), and $y_{j}$ denotes the actual observed number of mortalities in the database (numerator). Variation in the theoretical probability of mortality was modeled by assuming the log odds were normally distributed. Thus the model is as follows:

$$
\begin{gathered}
\log \left(\pi_{j} /\left[1-\pi_{j}\right]\right)=\eta_{j} ; \\
\eta_{j} \stackrel{\text { ind }}{\sim} N\left(\mu, \sigma^{2}\right),
\end{gathered}
$$

where $\mu$ and $\sigma^{2}$ denote the unknown mean and variance, respectively, of the assumed normal random effects distribution. Parameters of the model were estimated in a Bayesian framework using WinBUGS software. A vague (noninformative) prior distribution was chosen for the parameters $\mu$ and $\sigma^{2}$. The 


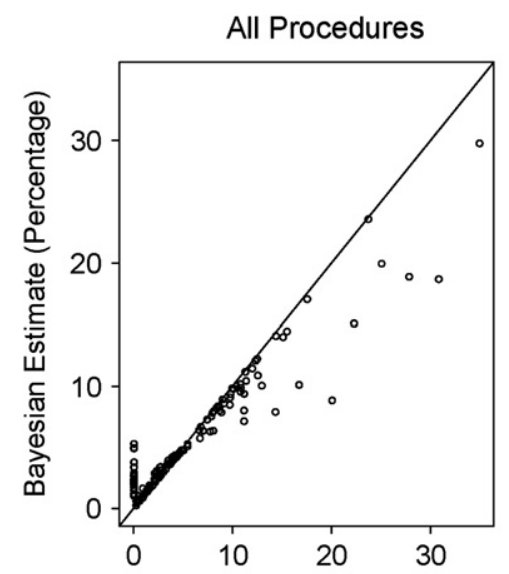

A Observed Mortality (Percentage)

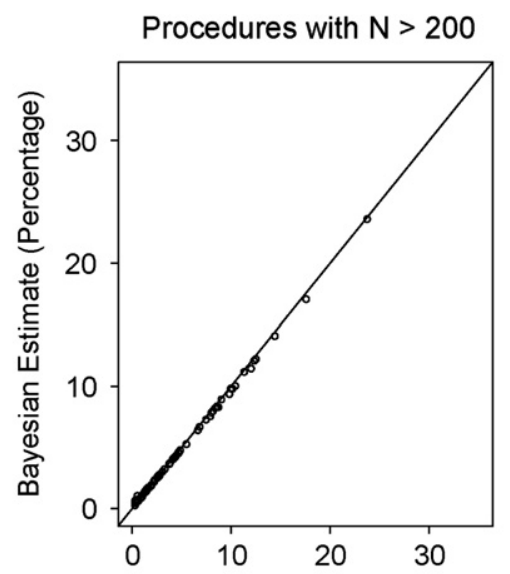

B Observed Mortality (Percentage)

FIGURE 6. Relationship between Bayesian model-based estimates and unadjusted mortality rates for individual procedures in the development sample.

WinBUGS code for this model is available from the authors on request.

As shown in Figure 6, A, there was a high degree of correlation between the Bayesian model-based estimate of a procedure's risk and the simple raw unadjusted mortality percentage; however, several procedures had

\section{Appendix 2. Methodology for Creating Internally Homogeneous Risk Categories}

Procedures were first sorted in order of increasing estimated risk (based on the model in Appendix 1) and then grouped into homogeneous categories to create the risk categories. Let $\pi_{i}$ denote the true unknown mortality for the $i$-th procedure, and let $\widehat{\pi}_{i}$ denote the corresponding estimate. We first sorted procedures so that $\widehat{\pi}_{1}<\widehat{\pi}_{2}<\cdots<\widehat{\pi}_{148}$. Let $k$ denote the number of categories and let $\boldsymbol{c}_{k}=\left\{c_{1}<c_{2}<\cdots<c_{k-1}\right\}$ denote a set of category cut points that partition the categories into $k$ groups. The symbol $c_{j}$ denotes a number between 1 and 148 and represents the index of the highest-risk procedure in the $j$-th category. Also, define $c_{0}=0$ and $c_{k}=149$. For any particular choice of $k$ and $\boldsymbol{c}_{k}$, within-category homogeneity is measured by the weighted sum-of-squares criterion:

$$
\operatorname{WSS}\left(\boldsymbol{c}_{k} ; \pi\right)=\sum_{j=1}^{k} \sum_{i=c_{j-1}+1}^{c_{j}} \frac{n_{i}\left(\pi_{i}-\bar{\pi}_{j}\right)^{2}}{\pi_{i}\left(1-\pi_{i}\right)},
$$

where $\bar{\pi}_{j}=\sum_{i=c_{j-1}+1}^{c_{j}} n_{i} \pi_{i} / \sum_{i=c_{j-1}+1}^{c_{j}} n_{i}$ denotes the average risk of mortality among all procedures in the $j$-th category. This criterion is similar to one that has been used previously for defining optimum cut points for categorizing a continuous explanatory variable. ${ }^{9}$ The notation $\operatorname{WSS}\left(\boldsymbol{c}_{k} ; \pi\right)$ is intended to emphasize that WSS is a function of the chosen cut points $\boldsymbol{c}_{k}$ and also depends on the unknown procedurespecific probabilities $\pi_{i}$. If the $\pi_{i}$ were known instead of unknown, then the "optimal" cut points could (in theory) large discrepancies. The difference between the modelbased versus raw estimates decreased with increasing sample size. For procedures with more than 200 cases, the raw and model-based estimates were virtually identical (Pearson correlation coefficient > 0.999; Figure 6).

be determined by enumerating all possible choices for the $c_{j}$ and choosing the one that minimizes the WSS. Because the $\pi_{i}$ are unknown, we instead choose cut points that minimize the Bayesian estimate of WSS $\left(\boldsymbol{c}_{k} ; \boldsymbol{\pi}\right)$. Specifically, we chose the cut points that minimize the estimated Bayesian posterior mean as follows:

$$
\widehat{\mathrm{WSS}}\left(\boldsymbol{c}_{k}\right)=\frac{1}{3000} \sum_{h=1}^{3000} \operatorname{WSS}\left(\boldsymbol{c}_{k} ; \pi^{(h)}\right),
$$

where $\pi^{(h)}$ denotes a random draw from the joint posterior distribution of the $\pi_{i}$ 's. Finding the set of cut points that minimizes this quantity exactly is technically challenging and required the use of a novel dynamic programming algorithm (unpublished).

The criterion described above gets smaller as the withincategory homogeneity improves. For plotting the change in homogeneity versus $k$, it is intuitively appealing to use a criterion that increases rather than decreases. The criterion used in Figure 2 (and throughout the article) is defined as follows:

$$
\text { Homogeneity }=1-\widehat{\mathrm{WSS}}\left(\boldsymbol{c}_{k}\right) / \widehat{\mathrm{WSS}}\left(\boldsymbol{c}_{1}\right) .
$$

This criterion ranges from 0.0 to 1.0 and increases as the categories become more homogeneous. 


\section{Appendix 3. Completeness of STS Mortality Data}

The mortality end point for this study was mortality status at the time of discharge, ie, in-hospital mortality. It was chosen over operative mortality (ie, death prior to discharge or after discharge but within 30 days of surgery) or 30-day mortality status in large part because 30-day status is frequently missing whereas discharge mortality is rarely missing. As shown in Figure 7, the completeness of 30-day mortality status has improved over time. In the future, it may be feasible to adapt the STS-EACTS methodology (or develop a new methodology) to predict the endpoint of operative mortality or 30-day mortality, assuming the completeness of 30-day mortality reporting continues to improve.

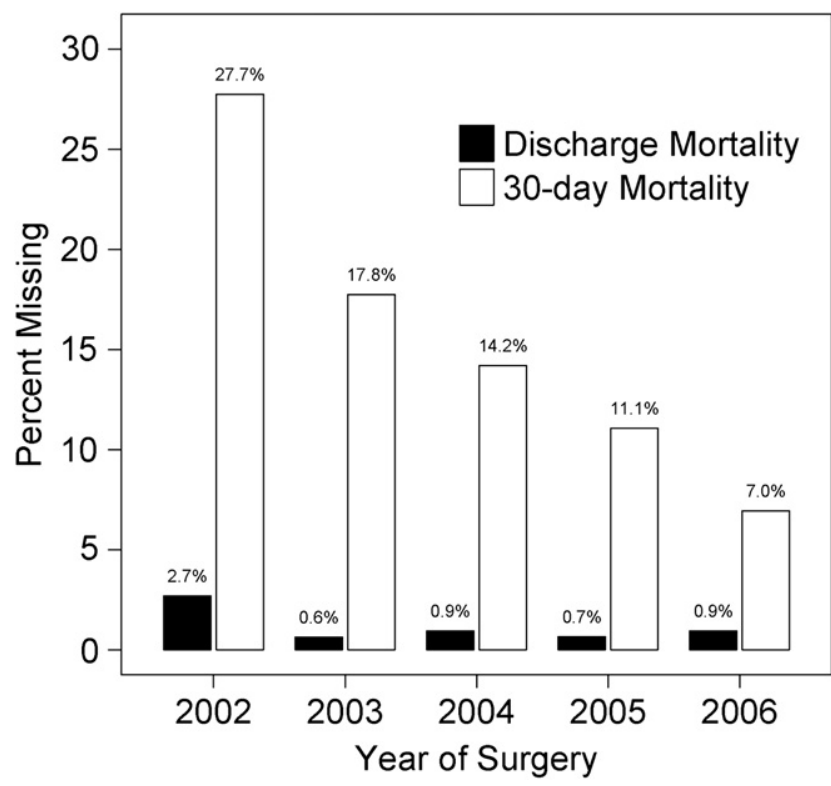

FIGURE 7. Decreasing percentage of missing data in the fields "mortality discharge status" (alive or dead) and "status at 30 days after surgery", (alive, dead, or unknown) in the Society of Thoracic Surgeons Congenital Database from 2002 to 2006. 